\title{
Artificial Synaptic Conductances Reduce Subthreshold Oscillations and Periodic Firing in Stellate Cells of the Entorhinal Cortex
}

\author{
Fernando R. Fernandez and John A. White \\ Department of Biomedical Engineering, Boston University, Boston, Massachusetts 02115
}

\begin{abstract}
Previous work has established that stellate cells of the medial entorhinal cortex produce prominent intrinsic subthreshold oscillations in the voltage response concentrated within the theta range $(3-7 \mathrm{~Hz})$. It has been speculated that these oscillations play an important role in vivo in establishing network behavior both in the entorhinal cortex and hippocampus. Consequently, it is important to investigate under what conditions theta oscillations in stellate cells can be generated and whether the spike-train power spectral density (PSD) also carries power at theta. We investigated the ability of stellate cells to generate theta oscillations in the presence of generic in vivo-like patterns of stimulation. Inputs were Poisson process-driven excitatory and inhibitory synaptic conductances or currents, introduced via dynamic clamp. We analyzed the subthreshold membrane oscillations and spike-train behavior in the presence of comparable synaptic conductance- or current-mediated membrane fluctuations. In the presence of conductance-based synapses, subthreshold oscillations are highly attenuated or entirely eliminated. Conversely, with current-based synapses stellate cells retain their ability to generate subthreshold oscillations in the theta band. These results also extend into the spiking regime, where only under current-based synapses does the PSD of the spike train show a prominent peak at theta. Furthermore, the peak in the spike-train PSD and spike clustering results from an increased probability of firing after a spike afterhyperpolarization and not directly from subthreshold oscillatory dynamics as has been previously suggested. Our results suggest that subthreshold oscillations may contribute less to in vivo response properties than has been hypothesized.
\end{abstract}

Key words: membrane conductance; medial entorhinal cortex; theta; oscillations; dynamic clamp; AHP

\section{Introduction}

It is well established that central neurons in vivo are continuously bombarded with thousands of synaptic inputs (Softky and Koch, 1993; Anderson et al., 2000; Destexhe et al., 2001, 2003). In many cases the activity of neurons in vivo is caused by the ongoing, and seemingly random activation of synapses (Softky and Koch, 1993; Pare et al., 1998; Shadlen and Newsome, 1998; Anderson et al., 2000; Destexhe et al., 2003). Input resistance measurements made in vivo have observed lower values compared with in vitro recordings (Borg-Graham et al., 1998; Pare et al., 1998; Destexhe et al., 2003). It has been suggested that neurons in vivo are under a high conductance state established by a balance between excitatory and inhibitory synaptic inputs (van Vreeswijk and Sompolinsky, 1996; Chance et al., 2002; Berg et al., 2007). Hence, neurons in vivo can, potentially, experience a large change in their

Received Dec. 20, 2007; revised Feb. 5, 2008; accepted March 1, 2008.

This work was supported by a postdoctoral fellowship from the Canadian Institutes of Health Research (F.R.F.) and grants from the National Institutes of Health (J.A.W.). We thank W. Hamish Mehaffey, Erwin Idoux, L. E. Moore, Tilman J. Kispersky, Michael N. Economo, and Kyle P. Lillis for useful discussions and comments on this manuscript. Correspondence should be addressed to Fernando R. Fernandez, Department of Bioengineering, University of Utah, 108 BPRB, 20 South 2030 East, Salt Lake City, UT 84112. E-mail: f.fernandez@utah.edu.

F. R. Fernandez's and J. A. White's present address: Department of Bioengineering, University of Utah, Salt Lake City, UT 84112 .

D01:10.1523/JNEUROSCI.5658-07.2008

Copyright $\odot 2008$ Society for Neuroscience $\quad$ 0270-6474/08/283790-14\$15.00/0 spatiotemporal integration scale and spiking behavior because of background synaptic activity (Bernander et al., 1991; Softky and Koch, 1993; Shadlen and Newsome, 1998; Wolfart et al., 2005)

In the present work, we studied the effects of in vivo-like conditions on the intrinsic properties of entorhinal cortical stellate cells. Slice recordings of stellate cells are characterized by subthreshold oscillations within the theta range (Alonso and Llinas, 1989). These oscillations have been assumed to play an important role in models of entorhinal network activity (O'Keefe and Burgess, 2005; Burgess et al., 2007; Giocomo et al., 2007). There are as yet no intracellular recordings from stellate cells in vivo to evaluate the role of intrinsic dynamics. Morphological data show that these cells receive thousands of inputs over the somatic-dendritic axis originating both outside and within the entorhinal cortex (Kohler, 1986; Swanson and Kohler, 1986; Gloor, 1997; Klink and Alonso, 1997), suggesting that stellate cells can experience changes in membrane conductance similar to those observed in other cortical regions. Single-unit statistics of layer II in vivo under anesthesia indicate that cells generate a peak in the power spectrum at theta (Alonso and Garcia-Austt, 1987b; Dickson et al., 1995), although similar recordings in behaving animals have no peak in the power spectrum (Frank et al., 2001). It remains unclear whether intrinsic stellate cell properties contribute to the network oscillatory activity observed in vivo, and how in vivo conditions alter the expression of intrinsic oscillatory properties. 
Using dynamic clamp, we stimulated stellate cells with an in vivo-like stimulus consisting of Poisson distributed synaptic conductance or current inputs. The expression of theta oscillations in the subthreshold membrane and spike-train response is eliminated with the introduction of a synaptic conductance stimulus, while a comparable synaptic current stimulus preserves the expression of the oscillations. Conductance-based stimulus abolished theta oscillations in the subthreshold and spike train using a different biophysical mechanism, indicating that the dynamics underlying the expression of subthreshold and spike-train periodicity are different. We find that the expression of oscillations in the spike train is dependent on the spike afterhyperpolarization (AHP), and not directly on subthreshold oscillations.

\section{Materials and Methods}

Tissue preparation. All experimental protocols were approved by the Boston University and University of Utah Institutional Animal Care and Use Committees. Horizontal sections of hippocampus and entorhinal cortex were prepared from 19- to 31-d-old Long-Evans rats. All chemicals were obtained from Sigma (St. Louis, MO) unless otherwise noted. After anesthetization with isoflurane and euthanasia, brains were removed and immersed in $0^{\circ} \mathrm{C}$ artificial CSF (ACSF) consisting of the following (in $\mathrm{mm}$ ): $125 \mathrm{NaCl}, 25 \mathrm{NaHCO}_{3}, 25$ D-glucose, $2 \mathrm{KCl}, 2 \mathrm{CaCl}_{2}, 1.25$ $\mathrm{NaH}_{2} \mathrm{PO}_{4}, 1 \mathrm{MgCl}_{2}$, and buffered to $\mathrm{pH} 7.4$ with $95 / 5 \% \mathrm{O}_{2} / \mathrm{CO}_{2}$. Horizontal slices were cut to a thickness of $400 \mu \mathrm{m}$ (Vibratome 1000+; Vibratome, St. Louis, MO). Slices were incubated in a $32^{\circ} \mathrm{C}$ bubbled ACSF for $30 \mathrm{~min}$ before being cooled to room temperature $\left(20^{\circ} \mathrm{C}\right)$. After the incubation period, slices were moved to the stage of an infrared, differential interference contrast-equipped microscope (Axioscope 2+; Zeiss, Oberkochen, Germany). Slices were bathed in ACSF with $10 \mu \mathrm{M}$ CNQX, $50 \mu \mathrm{M}$ picrotoxin, and $30 \mu \mathrm{M}$ AP- 5 to block ionotropic synaptic activity. All recordings were conducted between 32 and $34^{\circ} \mathrm{C}$.

Electrophysiology. Electrodes were drawn on a horizontal puller (P97; Sutter Instruments, Novato, CA) and filled with an intracellular solution consisting of the following (in mM): $120 \mathrm{~K}$-gluconate, $20 \mathrm{KCl}, 10 \mathrm{HEPES}$, 7 diTrisPhCr, $4 \mathrm{Na}_{2} \mathrm{ATP}, 2 \mathrm{MgCl}_{2}, 0.3$ Tris-GTP, and 0.2 EGTA, buffered to $\mathrm{pH} 7.3$ with $\mathrm{KOH}$. Final electrode resistances were between 3 and 4 $\mathrm{M} \Omega$, with access values between 4 and $12 \mathrm{M} \Omega$. All recordings were taken from the medial entorhinal cortex (MEC). Stellate cells were identified as neurons within layer II of the MEC exhibiting a large sag profile in response to hyperpolarizing current and having a peak between 3 and 7 $\mathrm{Hz}$ in the power spectral density of the subthreshold voltage response. Electrophysiological recordings were performed with a current-clamp amplifier (Axoclamp 2A; Molecular Devices, Union City, CA), and data were acquired using custom software developed in Matlab (MathWorks, Natick, MA) using the data acquisition toolbox.

For dynamic-clamp experiments, the current-clamp amplifier was driven by an analog signal from an $\times 86$ personal computer running an updated version of real-time application interface Linux and the realtime Linux dynamic clamp (Dorval et al., 2001) called Real-Time Experimental Interface. For all experiments, the dynamic clamp was set between 5 and $10 \mathrm{kHz}$. A measured junction potential of $\sim 10 \mathrm{mV}$ was subtracted from all recordings and taken into account during dynamicclamp experiments.

For all experiments, except those presented in Figure 3, data were collected at $2.5 \mathrm{kHz}$ and filtered at $1 \mathrm{kHz}$. For information presented in Figure 3 data were collected at $10 \mathrm{kHz}$ and filtered at $3 \mathrm{kHz}$.

Protocols. Synaptic protocols consisted of two independent Poisson processes generating unitary synaptic events. Individual synaptic events were modeled using a biexponential function. For inhibition the rise and decay time constants were 0.5 and $5 \mathrm{~ms}$, respectively, whereas for AMPAbased excitation these values were 0.25 and $2.5 \mathrm{~ms}$. For the NMDA-based excitatory synapse used in supplemental Figure 1 (available at www. jneurosci.org as supplemental material), a rise and decay time constant of 5 and $150 \mathrm{~ms}$ was used, respectively. The voltage dependence associated with $\mathrm{Mg}^{2+}$ block was modeled as follows:

$$
G_{\mathrm{NMDA}}(V)=\frac{1}{1+e^{\left(\left(V-V_{\text {half }}\right) l-k\right)}}\left(V_{\text {half }}=-10, k=12\right) .
$$

Note that these values are identical to previous implementations of NMDA using dynamic clamp and are based on experimental data (Harsch and Robinson, 2000).

The reversal value for excitation was set to $0 \mathrm{mV}$, whereas that for inhibition was $-75 \mathrm{mV}$. Note, these values are consistent with previous measurements of synaptic reversals in MEC stellate cells (Jones, 1994). For excitation and inhibition, individual synaptic events had the same maximal conductance, which ranged from $0.9-1.2 \mathrm{nS}$ for different cells (Netoff et al., 2005). In experiments where both AMPA and NMDA were used (supplemental Fig. 1, available at www.jneurosci.org as supplemental material), the maximal conductance was split evenly between AMPA and NMDA. For current-based synaptic protocols the reversal term was not included and maximal current for individual events was set between 12 and $15 \mathrm{pA}$. Note, individual maximal conductance or current for simulated synapses was slightly varied to produce the same fluctuation sizes (as determined by the SD of the membrane response) in different cells. Under control conditions, stellate cells remained quiescent with a resting membrane potential of approximately $-75 \mathrm{mV}$. The addition of the synaptic conductance and current stimuli provided a net depolarization $(\sim 15 \mathrm{mV})$ and hyperpolarization $(\sim 5 \mathrm{mV})$, respectively. Under all cases with synaptic-based stimuli, direct current (DC) was used to maintain cells at the same mean membrane voltage and firing frequency.

Inhibitory and excitatory events were delivered at a frequency of 1000 and $500 \mathrm{~Hz}$, respectively. The frequency of excitatory and inhibitory synapses was based on the fact that spontaneous background inhibitory frequency is greater than excitatory frequency in slices (Jones and Woodhall, 2005). Furthermore, a higher rate of inhibition was required to balance the membrane voltage near threshold. Hence, inhibition had to be presented at a higher rate than excitation to prevent the cells from firing at very high frequencies. Given the kinetics chosen for the synaptic conductances, the rates of 500 and $1000 \mathrm{~Hz}$ provided an in vivo-like level of membrane fluctuation size (Pare et al., 1998; Destexhe and Pare, 1999; Destexhe et al., 2003)

For the AHP subtraction experiments using dynamic clamp, we simulated an AHP-subtracting current $\left(I_{-\mathrm{AHP}}\right)$ using a voltage-dependent gating and time-constant variable modeled as $I=-g_{\max } x\left(V_{\text {membrane }}+\right.$ $90)$, with $x$ obeying $\frac{d x}{d t}=\frac{x_{\infty}-x}{\tau(V)}$ with $x_{\infty}$ defined as $\frac{1}{1+e^{\left(\left(V-V_{\text {hall }} /-k\right)\right.}}$ $\left(V_{\text {half }}=-25, k=3\right)$ and $\tau(V)$ defined as $\frac{100}{e^{\left(V_{\text {membrane }}+50\right) / 2}}-e^{\left(V_{\text {menbrane }}+6\right) /-50}$ +10 . For experiments where the AHP was increased in size $\left(I_{+A H P}\right)$, the current sign was inverted with steady-state values remaining the same. The function describing $\tau(V)$ was slightly slowed and described as follows: $\frac{130}{e^{\left(V_{\text {membrane }}+50\right) / 2}}$ $-e^{\left(V_{\text {membrane }}+6\right) /-50}+10$. The use of a voltage-dependent gating and time-constant variable permitted precise control over the shape of the resulting AHP.

Data analysis. All analysis was performed in Matlab using built in functions and/or custom software. For subthreshold conditions (with or without synaptic inputs) $50 \mathrm{~s}$ duration sweeps were used to generate power spectral density (PSD) plots for individual cells at a frequency resolution of $0.2 \mathrm{~Hz}$. For synaptic driven spike trains $500 \mathrm{~s}$ duration sweeps were used to generate PSD plots at a frequency resolution of 0.12 Hz. Finally, for spike trains in the absence of synaptic inputs $300 \mathrm{~s}$ duration sweeps were used to generate PSD plots at a frequency resolution of $0.3 \mathrm{~Hz}$. All PSD plots were calculated using the psd function in Matlab. Power within specified frequency ranges was determined as the integral of the PSD within the frequency range noted. Impedance was measured using a current input consisting of filtered zero-mean Gaussian noise (50 s duration and with a cutoff frequency of $150 \mathrm{~Hz}$ ) and taking the ratio of the Fourier transforms of the membrane voltage response and current input. Spikes were detected using the first derivate of the voltage trace, with threshold defined as a voltage derivate value 5 SDs away from the mean baseline value. Significance was determined using either a twosample unpaired Student's $t$ test or a one-way ANOVA using Tukey's 
honestly significant difference criterion. Means are presented with the SEM.

\section{Results}

Stellate cells produce subthreshold and perithreshold oscillations in the theta band of frequencies, defined here as 3 to $7 \mathrm{~Hz}$, ranging in amplitude from 2 to $6 \mathrm{mV}$ (Alonso and Llinas, 1989; Alonso and Klink, 1993; Klink and Alonso, 1993; Giocomo et al., 2007) (Fig. 1 A). A PSD analysis of subthreshold membrane voltage traces (50 s duration) produced a prominent peak at theta when cells were held between -63 and $-58 \mathrm{mV}$. The peak in the PSD occurred at $4.5 \pm 0.5 \mathrm{~Hz}$ (Fig. $1 A)(n=10)$.

To assess the impact of continuous synaptic activity on MEC stellate cell activity, we used the dynamic-clamp technique to artificially introduce conductance changes associated with EPSPs and IPSPs. We constructed synaptic conductance stimuli consisting of two independent Poisson processes that generated excitatory (EPSGs) and inhibitory postsynaptic conductances (IPSGs). We based the individual synaptic events in the stimulus protocol on previous intracellular work in stellate cells, which has quantified both the range in conductance and kinetics of AMPA and $\mathrm{GABA}_{\mathrm{A}}$ synaptic inputs (Jones, 1994; Berretta and Jones, 1996; Jones and Woodhall, 2005; Netoff et al., 2005). We set the EPSG and IPSG mean rates to 500 and $1000 \mathrm{~Hz}$, respectively, and used an identical maximum conductance for excitatory and inhibitory PSGs ranging from 0.9-1.2 nS. We used a double exponential function to describe the kinetics of individual PSGs, with faster kinetics describing EPSGs than IPSGs (see Materials and Methods).

To understanding how conductance inputs uniquely impact intrinsic neuronal properties, we compared the stellate cell output under the synaptic conductance stimulus with the output driven by an analogous synaptic current stimulus. In the case of the current stimulus, we used a maximum current for individual synaptic events between 12 and $15 \mathrm{pA}$, and set other variables to be equal to those of the conductance stimulus. We set the magnitude of both the synaptic conductance and current stimuli such that the stellate cell membrane voltage produced fluctuations with $\sim 10 \mathrm{mV}$ peak to peak amplitudes. Accordingly, the synaptic conductance and current protocols produced subthreshold membrane voltage fluctuations with SDs of $3.2 \pm 0.2 \mathrm{mV}(n=6)$ and $3.14 \pm 0.14 \mathrm{mV}(n=6)$, respectively, when held between -65 and $-68 \mathrm{mV}$. These values are comparable with what has been measured in vivo in other cortical neurons (Pare et al., 1998; Destexhe et al., 2003).

In stellate cells the introduction of a synaptic conductance stimulus abolished the characteristic peak at theta in the PSD of subthreshold voltage traces (Fig. $1 B$, black squares). Under the synaptic current stimulus, which generated a comparable level of membrane voltage fluctuations, the PSD retained its peak at theta, with maximum power occurring at $3.6 \pm 0.3 \mathrm{~Hz}(n=14)$. As a result, membrane voltage traces under the synaptic current stimulus.

\section{control}

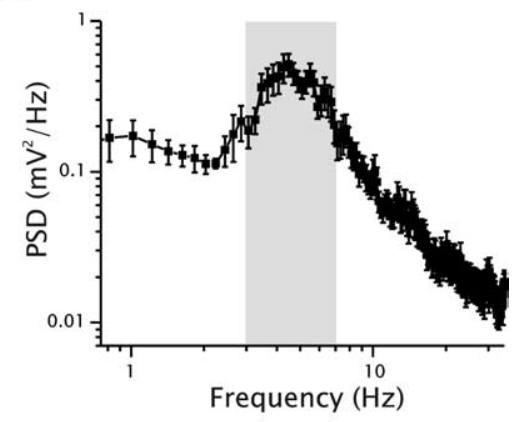

with synaptic inputs

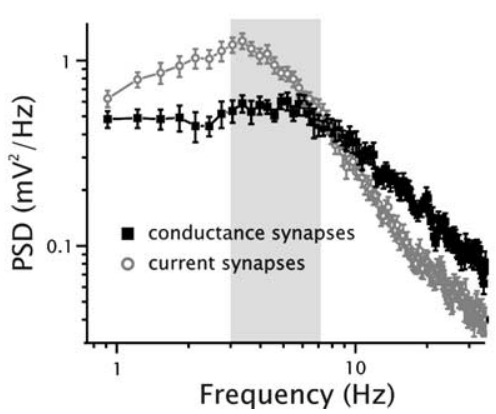

Figure 1. Elimination of subthreshold theta oscillations in stellate cells in the presence of a synaptic conductance stimulus. $\boldsymbol{A}$, Note the decreased power in the membrane voltage response within the theta range $(3-7 \mathrm{~Hz})$ with the conductance-based

stimulus had significantly more power within the theta range $(p<0.05, n=14)$. Another difference between the synaptic conductance and current stimuli was the amount of power in the PSD at high frequencies (Fig. $1 B$ ). Under the synaptic conductance stimulus, the PSD had significantly more power at frequencies $>10.0 \mathrm{~Hz}$ (power calculated between 10.0-35 Hz; $p<0.05$, $n=14$ ).

Although the synaptic current stimulus preserved the peak in the PSD, the frequency at which it occurred was significantly lower than in the control case $(3.6 \mathrm{~Hz}$ vs $4.5 \mathrm{~Hz}, p<0.05, n=14)$. To account for this difference it is important to consider that preventing the cell from firing under the synaptic-mediated fluctuations requires significant hyperpolarization compared with control. This is because of the lowering of spike rheobase (input current required to elicit spike firing) and smoothing of the frequency-current relationship under noisy conditions (Barbi et al., 2000; Longtin, 2000; Chance et al., 2002; Miller and Troyer, 2002). The membrane fluctuations associated with background synaptic activity permit occasional threshold crossings despite maintaining a mean membrane voltage that is significantly more negative than that required to elicit spikes in the absence of noisy synaptic stimuli. As a consequence, the membrane voltage must be held at a more negative value to prevent spike firing under the synaptic stimulus conditions. This difference in mean holding voltage can account for the shift in the peak frequency of the PSD between the control and the synaptic stimulus scenario. Previous 
work has shown that subthreshold oscillatory frequency increases with depolarization by $\sim 0.2 \mathrm{~Hz} / \mathrm{mV}$ (Alonso and Klink, 1993; Giocomo et al., 2007), which is consistent with our observation of a $1 \mathrm{~Hz}$ change over $\sim 6 \mathrm{mV}$. Last, the peak of the PSD with synaptic currents was wider in the frequency axis compared with the control scenario. This effect was because of the noisy character of the synaptic stimulus.

In summary, the introduction of a Poisson distributed synaptic conductance stimulus was sufficient to abolish stellate cell intrinsic subthreshold oscillations. Conversely, under a synaptic current stimulus, stellate cells maintained the ability to generate theta oscillations.

\section{Modulation of theta oscillations in spike trains by synaptic conductance inputs}

In the next set of experiments, we were interested in the modulation of the spike-train PSD by synaptic conductance and current stimuli. Although the expression of theta oscillations in stellate cells is well characterized in the subthreshold regime, it has not been established whether these oscillations translate into an observable peak in the PSD of the spike train. Given the role that subthreshold oscillations have been attributed in models of network behavior in the entorhinal cortex (O'Keefe and Burgess, 2005; Burgess et al., 2007; Giocomo et al., 2007), it is important that the ability for the spike train to carry power at theta be evaluated. Spiking nonlinearities have the potential to significantly alter the expression of subthreshold events and its modulation by synaptic inputs. Subthreshold oscillations in stellate cells also have highly irregular amplitudes (White et al., 1998; Dorval and White, 2005), which may impede their translation into the spike train. To analyze the PSD of spike trains we turned spike events into a value of one, while setting the interspike period into a sequence of zeros. In this way, the spike train becomes a binary sequence containing no direct information about subthreshold membrane voltage dynamics (Bair et al., 1994; Koch, 1999).

We started by establishing the control PSD for spike trains in the absence of any synaptic stimulus. PSD plots were calculated from cells firing between 2 and $3 \mathrm{~Hz}$. This range of frequency was used because it is well below the maximum firing frequency of stellate cells and provides the ideal range for the formation of spike clusters (Alonso and Klink, 1993). Spike clustering has been suggested to represent the entrainment of regular spike firing by subthreshold oscillations, and hence represent the expression of the subthreshold oscillation within the spike train (Alonso and Klink, 1993). Finally, in vivo work from awake animals has indicated average firing frequencies between 2 and $3 \mathrm{~Hz}$ for singleunit recordings from the superficial entorhinal cortex (Frank et al., 2001).

Stellate cells were depolarized to $-58 \pm 2 \mathrm{mV}$, such that mean firing rate averaged over a $300 \mathrm{~s}$ sweep was $\sim 2.4 \mathrm{~Hz}$. Under these conditions stellate cells clustered their spikes with intracluster interspike interval (ISI) values centered in the theta range (Fig. $2 \mathrm{Ai}$ ). As a result, stellate cells produced a peak in the PSD of spike trains at $4.9 \pm 0.4 \mathrm{~Hz}$ (Fig. 2 Aii) $(n=15)$.

We followed by introducing the same synaptic conductance and current stimuli used to assess the PSD in the subthreshold regime. We set cell membrane voltage to $-64 \pm 1.8 \mathrm{mV}$ and $-66.1 \pm 1.9 \mathrm{mV}$ for the synaptic conductance and current stimuli, respectively, to maintain spike firing frequency at $2.4 \mathrm{~Hz}$ (over $500 \mathrm{~s}$ duration sweeps). Note that stellate cells did not generate spikes in this voltage range in the absence of the synaptic stimuli. For these sets of experiments we also compared the effect of a synaptic conductance and current stimuli on the PSD of entorhinal layer II/III pyramidal cells firing at the same average frequency as stellate cells. As established previously (Klink and Alonso, 1993), pyramidal cells are easily discernible from stellate cells because of the absence of both subthreshold oscillations and a hyperpolarization induced membrane sag (Fig. 2 B). Accordingly, the analysis of pyramidal cells offers a type of control from which to assess the unique character of stellate cell spiking behavior.

Similar to the subthreshold analysis, the observation of a peak in the PSD of the spike trains of stellate cells depended on the nature of the synaptic stimulus. Under the synaptic current stimulus, the PSD of the spike train produced a peak at theta, whereas with the synaptic conductance stimulus the peak was abolished. This resulted in significantly less power at theta with the synaptic conductance stimulus (Fig. $2 \mathrm{Di}$, black squares) $(p<0.05, n=$ 15). In pyramidal cells, neither the synaptic conductance nor the current stimulus produced a discernable peak in the PSD of spike trains (Fig. 2 Dii). Note, we also tested the effects of introducing an NMDA synapse into the conductance-based stimulus on the spike-train PSD of stellate cells (supplemental Fig. 1, available at www.jneurosci.org as supplemental material). NMDA-mediated synaptic activity has the potential to significantly alter the results generated by the synaptic conductance stimuli because of the voltage dependence associated with $\mathrm{Mg}^{2+}$ block. As shown in supplemental Figure 1 (available at www.jneurosci.org as supplemental material), however, the PSD of the spike train in the presence of the NMDA-based synapse (along with AMPA and $\mathrm{GABA}_{\mathrm{A}}$ ) continued to have less power within theta and was similar to the result presented in Figure $2 \mathrm{Di}$, which incorporated only AMPA and $\mathrm{GABA}_{\mathrm{A}}$ synapses (supplemental Fig. 1, black squares, available at www.jneurosci.org as supplemental material) $(p<0.05, n=8)$.

Although the peak in the PSD of stellate cells was present in both the subthreshold and spiking regimes with the synaptic current stimulus, it occurred at a significantly higher frequency in the PSD of spike trains $(4.7 \pm 0.2 \mathrm{~Hz}$ vs $3.6 \pm 0.3 \mathrm{~Hz}, p<0.05)$. This result suggests two possible scenarios. The first is that the aforementioned increase in subthreshold oscillation frequency with depolarization (and consequently with spike initiation) continues into the spiking regime. A second possibility is that the spiking dynamics influence the expression of theta at the level of the spike train and alter how subthreshold dynamics influence spike-train behavior. In support of the second possibility, both the control (Fig. 2 Aii) and the synaptic current-driven (Fig. 2Di) spike trains produced a peak at the same frequency in the PSD despite being held at different membrane voltages $(-58 \pm 2 \mathrm{mV}$ vs $-66.1 \pm 1.9 \mathrm{mV}, p<0.05)$. Hence, we would have expected the control scenario, which was not driven with synaptic inputs and was more depolarized, to express a significantly higher peak frequency in the PSD if subthreshold dynamics translate unaltered into the spiking regime. Minimally, this result indicates that spiking dynamics influence the expression frequency of subthreshold oscillations in the spike train by changing the voltage dependence in the oscillation frequency observed in the subthreshold regime.

Under synaptic current and conductance stimuli stellate cells generated nearly identical mean firing frequencies of $2.4 \pm 0.2 \mathrm{~Hz}$ and $2.4 \pm 0.1 \mathrm{~Hz}$, respectively $(p>0.05, n=15)$. This indicated that the peak in the PSD was not caused by the mean firing frequency, as this value was always significantly less than the peak frequency of the PSD. A dip in power at low frequencies was also observed in all PSD of spike trains. Previous work on spike-train 
PSD has shown that this phenomenon can be either because of oscillatory behavior in the spike train or the refractory period of the cell during spike generation near the maximal firing rate (Bair et al., 1994; Koch, 1999). Both these factors tend to regularize the spike train and drive it away from a Poisson process with a flat PSD. Notably, the PSD of stellate cell spike trains driven with the synaptic current stimulus had significantly less power at frequencies $<2.5$ $\mathrm{Hz}$ than with synaptic conductance inputs $(p<0.05, n=15)$. It seemed probable that differences in the spike-train PSD between conductance and current stimuli were caused by changes in the oscillation properties and not the changes in the refractory period, because cells were kept well below their maximum firing rate of $20 \mathrm{~Hz}$ (Klink and Alonso, 1997). Hence, with the synaptic current stimulus, when cells were more oscillatory, the PSD had less power at frequencies $<2.5 \mathrm{~Hz}$.

\section{Sensitivity of theta oscillations to steady-state changes in membrane input resistance}

Although stellate cell output differed under synaptic conductance and current stimuli, we did not understand the mechanism by which the conductance-based stimulus abolished the peak in PSD of the subthreshold membrane voltage and spike-train responses. Moreover, it was unclear whether the same biophysical mechanism was responsible for abolishing the peak in PSD of both the subthreshold and spiking response.

To begin to address the mechanism by which synaptic conductance and current stimuli produced different results in the PSD we used dynamic clamp to introduce an artificial leak conductance without the corresponding noise associated with randomly timed synaptic inputs. In this way we could separate the effects of the synaptic conductance stimulus into the conductance and noisy fluctuation components. We started by measuring the magnitude of change in input resistance produced in stellate cells by the synaptic conductance stimulus. Steady-state membrane input resistance was measured in the subthreshold range between -95 and $-70 \mathrm{mV}$ by averaging the last $0.7 \mathrm{~s}$ of the membrane voltage traces at different levels of current injection and fitting a line to the current-voltage relationship of the cell (Fig. $3 A, B$ ). The addition of the synaptic conductance stimulus resulted in a decrease of $38 \%$ in steady-state input resistance from $64.6 \pm 5.4$ $\mathrm{M} \Omega$ to $40.6 \pm 2.1 \mathrm{M} \Omega$ (Fig. $3 B, C$ ) (one-way ANOVA, $p<0.05$, $n=7)$. We should note that this magnitude of change in input resistance is relatively modest compared with what has been re-

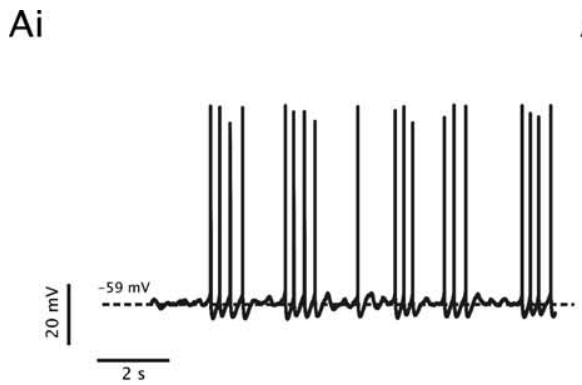

Aii

$\mathrm{Bi}$

Layer II stellate cell

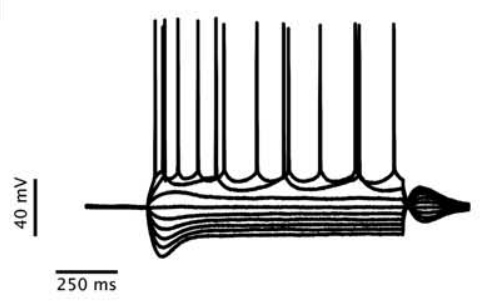

$\mathrm{Ci}$

conductance synapses current synapses
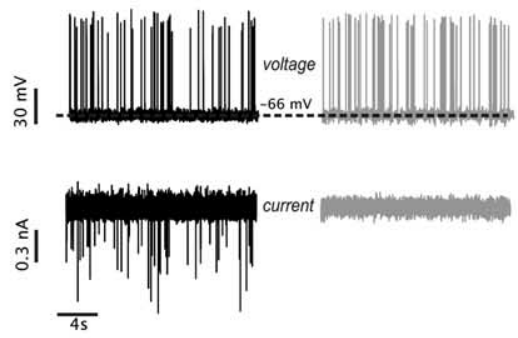

Bii

Layer II/III pyramidal cell

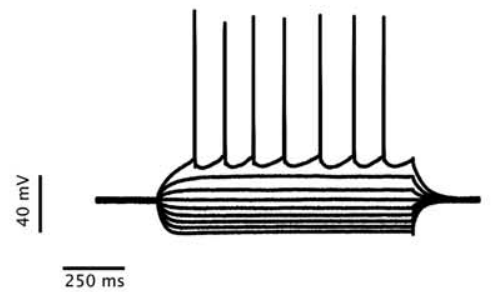

Cii

conductance synapses current synapses
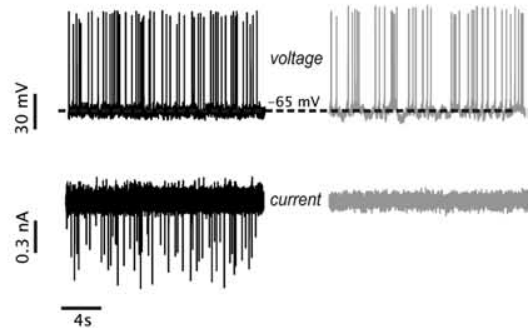

Dii

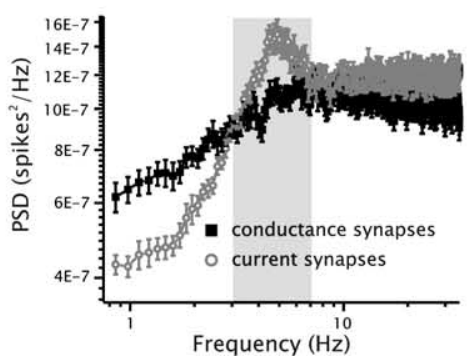

Figure 2. Expression of theta oscillations in the spike train of stellate cells is eliminated with a synaptic conductance stimulus. $A$, Average spike-train PSD of stellate cells under control conditions (no synaptic stimulus). At low firing frequencies $(2.4 \mathrm{~Hz})$, stellate cells clustered their spikes at interspike intervals within the theta range $(\sim 0.2 \mathrm{~s}$, left). Individual PSD curves were generated from 300 s duration sweeps and averaged across different cells (right). $\boldsymbol{B}$, Representative examples of stellate and layer II/III pyramidal cell responses to square current steps. Cells were held at $-75 \mathrm{mV}$ before delivery of $1 \mathrm{~s}$ duration square current steps, which ranged from $-0.3 \mathrm{nA}$ to $0.05 \mathrm{nA}$ in $0.05 \mathrm{nA}$ increments. Note that stellate cells are discernible from pyramidal cells because of the presence of a hyperpolarizing sag during the onset of the hyperpolarizing current step and an increased rebound in the membrane voltage response after cessation of the current step. $\boldsymbol{C}$, Representative voltage traces of stellate and pyramidal cell response to synaptic conductance- (black) and current-based (gray) stimuli. Note that the sharp transients in the conductance-based stimulus are caused by the changes in the driving force terms associated with spike generation. $\boldsymbol{D}$, Average spike-train PSD under conductance- (black squares) and current-based (gray circles) stimuli for stellate (Di) and layer II/III pyramidal cells (Dii). Note the decreased power in the spike train within the theta range with the conductance-based stimulus in stellate cells. Individual PSD curves were generated from 500 s duration sweeps and averaged across different cells.

ported for comparisons between in vitro and in vivo intracellular recordings, which can be as great as 80\% (Pare et al., 1998; Destexhe and Pare, 1999; Destexhe et al., 2003). To match the $38 \%$ decrease in input resistance, we introduced a single artificial leak conductance via dynamic clamp ranging in magnitude from 12 to 
$\mathrm{Ai}$ control

Aii

with synaptic conductance synapses
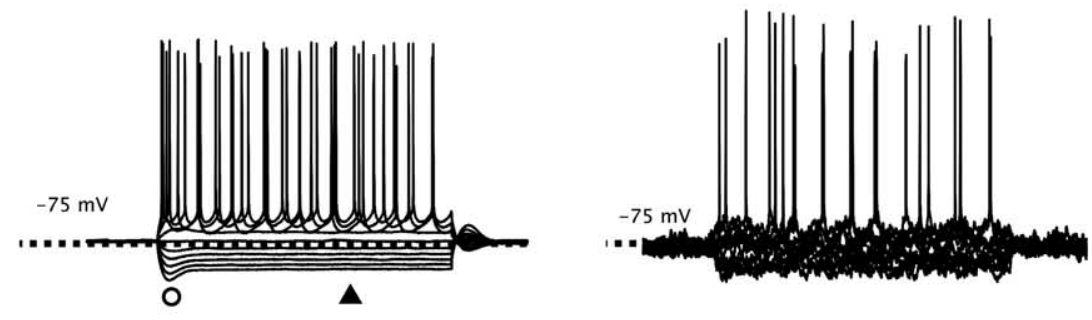

Aiii

with added leak conductance

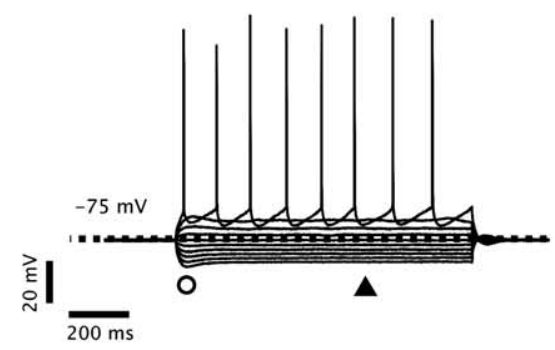

C

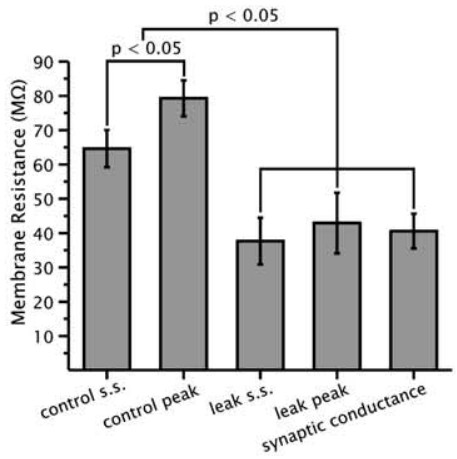

$\mathrm{E}$

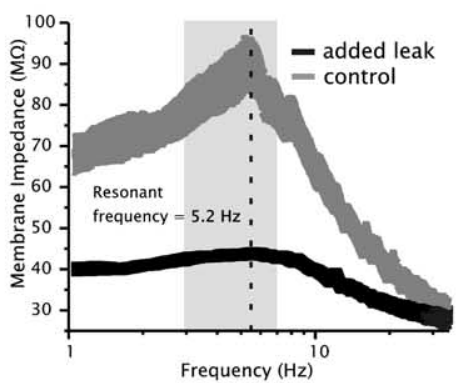

B

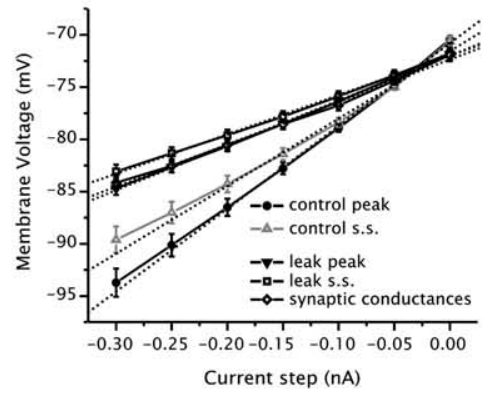

D

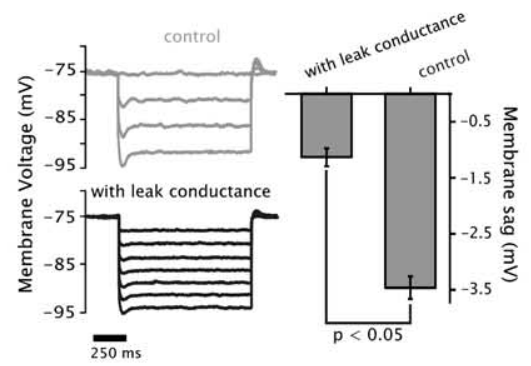

$\mathrm{F}$

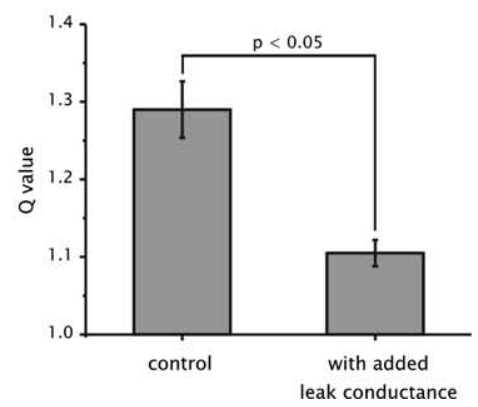

Figure 3. Introduction of an artificial leak conductance in stellate cells preferentially reduces voltage fluctuations within theta. $\boldsymbol{A}$, Representative traces of stellate cells in response to the same square current step protocol under control $(\boldsymbol{A} \boldsymbol{i})$, a synaptic conductances stimulus (Aii), and a 10 $-12 \mathrm{nS}$ artificial leak conductance (Aiii). Under all conditions, cells were held at $-75 \mathrm{mV}$ before delivery of $1 \mathrm{~s}$ duration square current steps, which ranged from $-0.3 \mathrm{nA}$ to $0.05 \mathrm{nA}$ in $0.05 \mathrm{nA}$ intervals. $\boldsymbol{B}$, Average current-voltage (I- $\eta$ relationship for peak and steady-state (s.s.) voltage response under control, the synaptic conductance stimulus, and the with the added leak conductance. The peak was measured individually for each trace, but corresponds approximately to the location of the circle in $\boldsymbol{A i}$ and $\mathbf{A i i i}$, whereas the steady-state is indicated by the black triangle. Dashed lines indicate linear fits to the average $I-V$ data under each specified condition. $\boldsymbol{C}$, Average input resistance $\left(R_{i}\right)$ for each condition outlined in $\boldsymbol{B}$. A linear fit $\left(r^{2}>0.98\right)$ of the $I-V$ relationship for individual cells was used to determine $R_{i}$ under each condition, which was then averaged for comparisons. D, Difference between peak and steady-state membrane voltage under control and with added leak conductance. The difference in membrane voltage (sag) was significantly greater in control (black traces) than with the added leak conductance (gray traces). Bar graph (right) indicates average sag amplitude measured between -87 and $-90 \mathrm{mV}$ in control and with the leak conductance. Cells were held at $-75 \mathrm{mV}$ before delivery of $1 \mathrm{~s}$ duration square current steps, which ranged from $-0.45 \mathrm{nA}$ to $0 \mathrm{nA}$ with leak and $-0.35 \mathrm{nA}$ to $0 \mathrm{nA}$ in control at $0.05 \mathrm{nA}$ intervals. $E$, Average impedance plot of stellate cells under control (gray line) and with the added leak conductance (black line). Line width denotes extent of error bars. $\boldsymbol{F}$, Ratio between peak (measured at $5.2 \mathrm{~Hz}$ ) and baseline $(1 \mathrm{~Hz})$ impedance ( $Q$ value) under control and with the added leak conductance. Under control conditions the $Q$ value was significantly greater than with the added leak conductance.

$15 \mathrm{nS}$, and with a reversal value of $-50 \mathrm{mV}$. The reversal value matched that observed under the synaptic conductance stimulus and was used for all experiments involving the addition of a leak conductance unless otherwise noted.

The introduction of the leak conductance decreased the membrane time constant from $11.4 \pm 0.9 \mathrm{~ms}$ to $7.5 \pm 0.4 \mathrm{~ms}$, as determined with a single exponential fit to a voltage step from -70 to $-75 \mathrm{mV}(n=$ $6)$. In the presence of the added leak conductance the steady-state input resistance was $37.7 \pm 6.8 \mathrm{M} \Omega$, which did not differ significantly from values measured in the presence of the synaptic conductance stimulus (Fig. $3 B, C$ ) (one-way ANOVA, $p>$ $0.05, n=7$ ). From the membrane voltage traces (Fig. $3 A$ ) it also seemed that the addition of the leak conductance reduced the hyperpolarization induced sag. To quantify this we compared the peak and steadystate input resistance between control and with the added leak conductance. Peak input resistance was measured using the voltage values from the transient peak associated with the hyperpolarization activated cation current $\left(I_{\mathrm{H}}\right)$. Under control conditions the peak and steady-state input resistance values were significantly different $(79.3 \pm 5.2 \mathrm{M} \Omega$ vs $64.6 \pm 5.4 \mathrm{M} \Omega$; oneway ANOVA, $p<0.05, n=7$ ), whereas with the added leak conductance the values were no longer significantly different (Fig. $3 B, C)(37.7 \pm 6.8 \mathrm{M} \Omega$ vs $42.9 \pm 8.8 \mathrm{M} \Omega$; one-way ANOVA, $p>0.05, n=7)$. This result suggested that changes in membrane conductance had a stronger effect on transient voltage changes than the steady state. To address this point more rigorously we compared the size of the membrane sag produced with similar sized voltage steps in control and with the added leak conductance. The membrane sag was measured as the voltage difference between the peak voltage transient and the steady-state membrane voltage. When cells were hyperpolarized between -87 and $-90 \mathrm{mV}$, the membrane sag was $3.46 \pm 0.22 \mathrm{mV}$ in control and $1.12 \pm 0.16 \mathrm{mV}$ with the added leak conductance (Fig. 3D) $(p<0.05$, $n=6)$.

We next compared the impedance profile of stellate cells under control and with the added leak conductance. Impedance was measured by holding cells between -72 and $-70 \mathrm{mV}$ and injecting them with a noisy current stimuli of 50 s duration (see Materials and Methods). For control and the leak modified cells we adjusted the amplitude of the current stimuli to maintain similar voltage fluctuations $(\sim 5-7 \mathrm{mV})$. Under control and with the added leak 

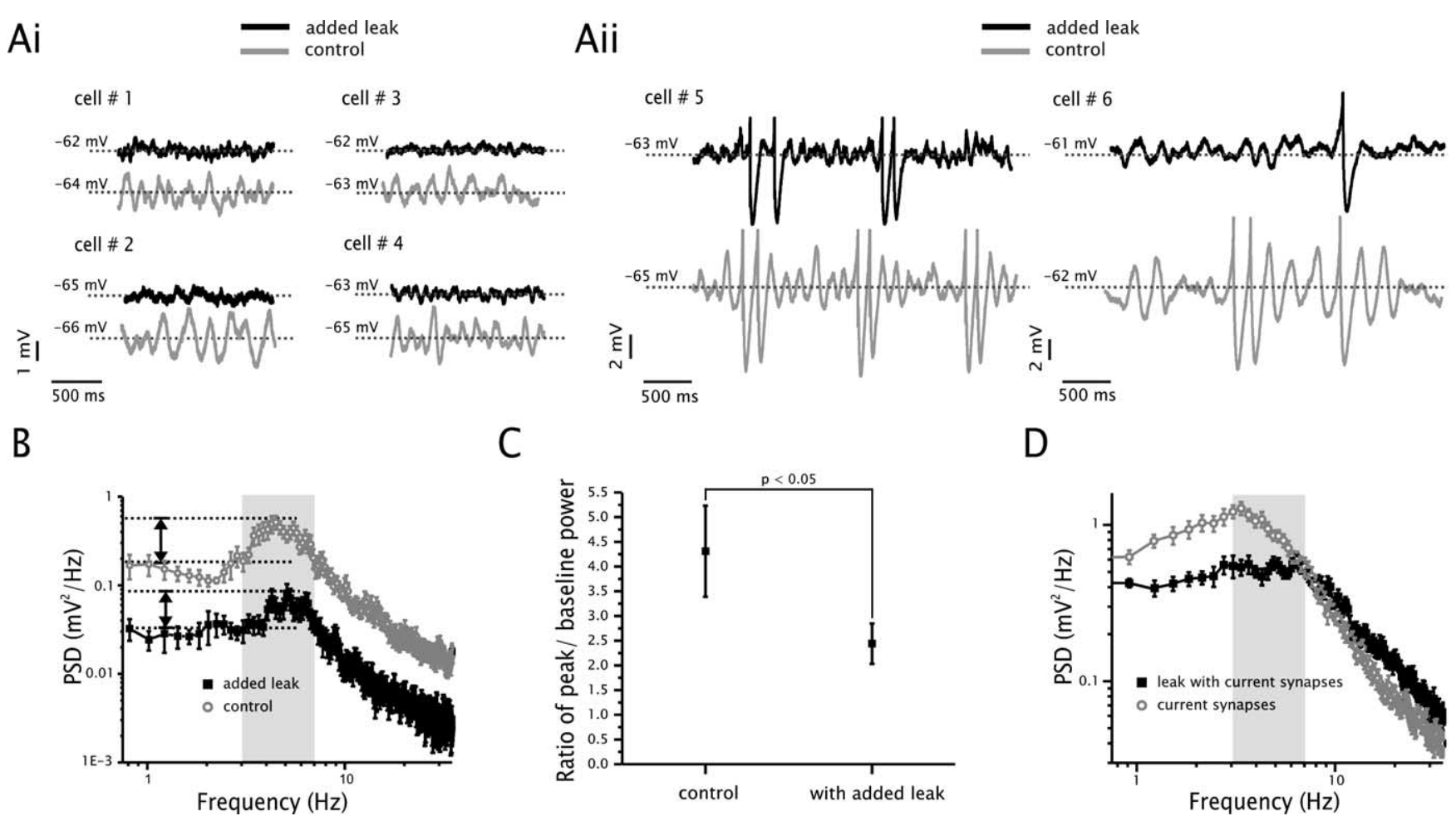

C

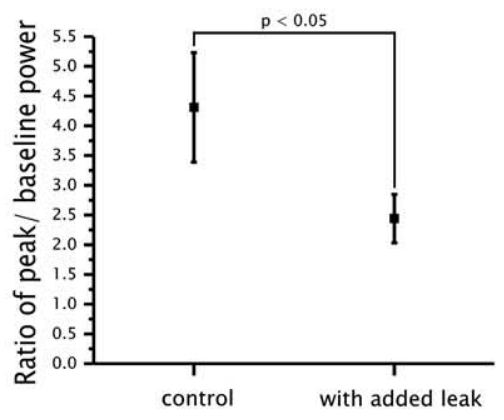

D

Figure 4. Introduction of a leak conductance significantly reduces the amplitude of subthreshold theta oscillations. $A$, Representative examples of stellate cell membrane voltage traces under control (gray) and with an added leak conductance (black) in the subthreshold (Ai) and low-frequency firing (Aii) regime ( $<1 \mathrm{~Hz})$. Note, in the presence of the leak conductance membrane voltage was held at a slightly more depolarized potential to account for the small change in firing threshold. $\boldsymbol{B}$, Average PSD of subthreshold voltage response under control (gray circles) and with added leak conductance (black squares). Dotted lines in $\boldsymbol{B}$ indicate difference in power between peak and baseline $(1 \mathrm{~Hz})$ power. $\boldsymbol{C}$, Ratio between peak and baseline $(1 \mathrm{~Hz})$ power of subthreshold response under control and with added leak conductance. $\boldsymbol{D}$, Average PSD of subthreshold voltage response in the presence of synaptic current stimuli with (black squares) or without an added leak conductance (gray circles). The amplitude of individual synaptic inputs was approximately doubled under the artificial leak conductance to maintain the same mean fluctuation size in the membrane response.

conductance the impedance profile of stellate cells had peak resonant frequencies of $5.2 \pm 0.1 \mathrm{~Hz}$ and $5.2 \pm 0.2 \mathrm{~Hz}$, respectively, which were not significantly different (Fig. $3 E)(p>0.05, n=8)$. The addition of the leak conductance, however, significantly reduced the magnitude of the resonance. A measurement of the $Q$ value (defined here as the ratio of the impedance at 5.2 and $1 \mathrm{~Hz}$ ) indicated that this value was $1.30 \pm 0.04$ under control and $1.11 \pm 0.02$ in the presence of the added leak conductance (Fig. $3 F)(p<0.05, n=8)$. Consistent with the membrane sag measurements, the impedance plots indicated that a reduction in membrane input resistance significantly reduced the resonant properties of stellate cells.

We proceeded to test the effects of adding a leak conductance on the expression of theta oscillations in the subthreshold membrane response. In most cells the addition of the leak conductance increased the voltage at which spikes were first observed by $\sim 1$ $\mathrm{mV}$. As a result, we depolarized cells with the added leak conductance by this amount for comparison with the control scenario. As shown in Figure $4 A$, the addition of the leak conductance (black traces) dramatically decreased the observation of theta frequency oscillations both in the subthreshold and lowfrequency spiking regimes compared with the control case (gray traces) when cells were held between -60 and $-65 \mathrm{mV}$. A comparison of the PSD plots of subthreshold voltage traces $(50 \mathrm{~s}$ duration) of the control and leak modified cells showed a significant decrease in power at theta in cells with the added leak conductance ( $p<0.05, n=11$ ). Although mostly diminished in the presence of the added leak conductance, a peak at theta in the PSD was still discernable at $4.3 \pm 0.39 \mathrm{~Hz}$ (Fig. $4 B$, black squares). Like the resonant frequency, the added leak conduc- tance did not significantly alter the frequency of the oscillations compared with control $(p>0.05, n=14, n=11)$.

To compare the size of the peak of the PSD relative to the baseline power (defined here as $1 \mathrm{~Hz}$ ) we calculated the average difference and ratio between the power at theta (at peak) and that at $1 \mathrm{~Hz}$. The difference in power was $0.25 \pm 0.05 \mathrm{mV}^{2} / \mathrm{Hz}$ in the control case, and only $0.032 \pm 0.01 \mathrm{mV}^{2} / \mathrm{Hz}$ in the presence of the added leak conductance ( $p<0.05, n=14$ and $n=11$ ). Moreover, the ratios between the peak and baseline power values in the control and with the added leak conductance were $4.3 \pm$ 0.9 and $2.4 \pm 0.4$, respectively (Fig. $4 C)(p<0.05, n=14, n=$ 11). Therefore, the introduction of a leak conductance reduced preferentially the magnitude of subthreshold membrane oscillations within the theta range.

We also looked at the PSD of the subthreshold response when both the added leak conductance and the synaptic current stimulus were present. We introduced a similar synaptic current stimulus as before, but with a larger size for individual synaptic events $(20 \mathrm{pA})$ to maintain a similar mean $(-64.8 \pm 1.1 \mathrm{mV})$ and SD $(-3.3 \pm 0.1 \mathrm{mV})$ in the membrane voltage response. Under the reduced membrane input resistance the peak in the PSD was abolished, with significantly less power in the theta range (Fig. $4 D)(p<0.05, n=8)$. Likewise, the PSD also showed significantly more power at frequencies $>10.0 \mathrm{~Hz}(p<0.05, n=8)$. Overall, the profile of the PSD with the added leak conductance and the synaptic current stimulus was very similar to the situation with the synaptic conductance stimulus alone (Fig. $1 B$, black squares). Therefore, the elimination of subthreshold theta oscillations with synaptic conductance inputs is likely a direct result of 

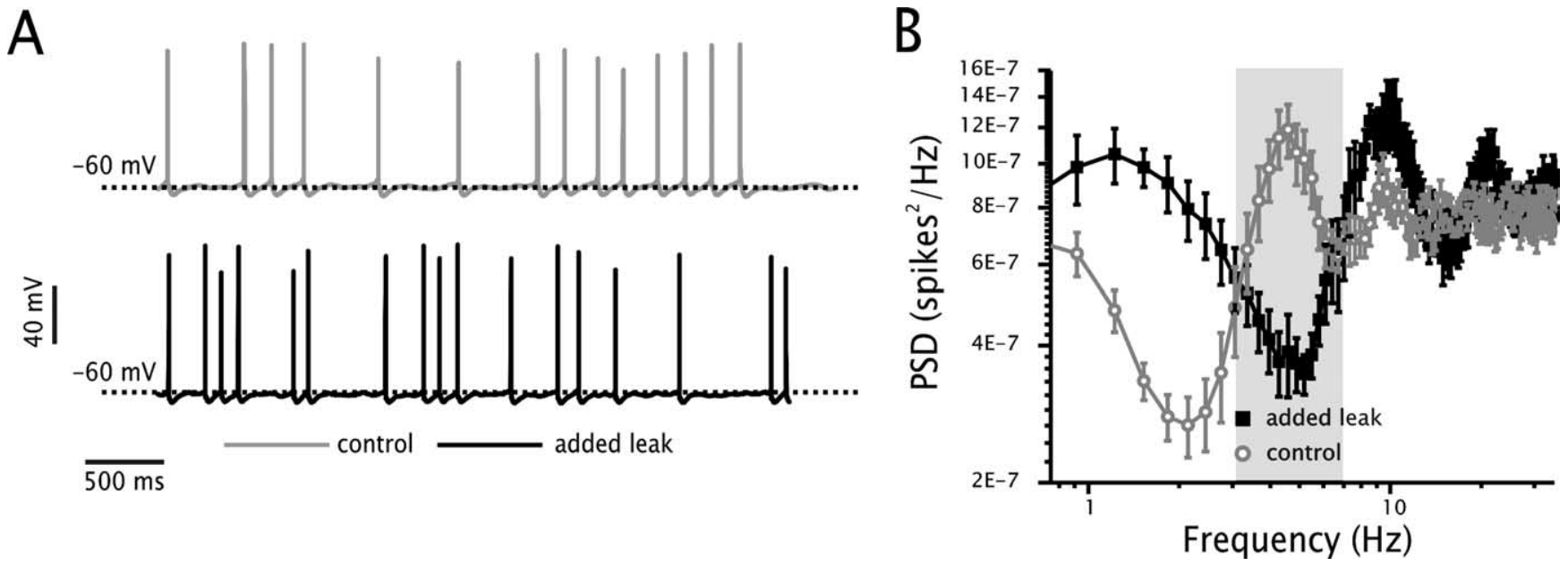

Figure 5. Introduction of a leak conductance increases the frequency within spike-train clusters. $A$, Representative voltage trace of a stellate cell under control conditions (gray) and with $13 \mathrm{nS}$ of added leak conductance (black). Note that the addition of the leak conductance preserved the spike clustering behavior but reduced the time interval between spikes. $\boldsymbol{B}$, Average spike-train PSD under control (gray circles) and with added leak conductance (black squares).

lower membrane impedance, which effectively reduces the resonant properties of the cell and dampens the oscillations.

We next asked whether the addition of the leak conductance could eliminate the peak at theta in PSD of the spike trains. Cells were depolarized to maintain a firing frequency of $\sim 2.4 \mathrm{~Hz}$ (over 300 s sweeps). Unlike the subthreshold membrane response, the presence of the added leak conductance (Fig. 5A, black trace) did not eliminate or diminish the peak in the PSD of the spike-train response. Rather, the peak frequency increased significantly compared with control (Fig. $5 A, B)(9.7 \pm 0.6 \mathrm{~Hz}$ vs $4.9 \pm 0.4 \mathrm{~Hz}, p<$ $0.05, n=9)$.

We next measured the PSD of the spike train in the presence of both the synaptic current stimulus and the added leak conductance ( $2.4 \mathrm{~Hz}$ over 500 s sweeps). Similar to the synaptic conductance situation, there was no discernable peak in the PSD of spike trains under these conditions (supplemental Fig. $2 A, B$, available at www.jneurosci.org as supplemental material) $(p<0.05, n=$ $8)$, with the added leak conductance also increasing the power in the PSD at frequencies $<2.5 \mathrm{~Hz}$ (Fig. $5 B, D)(p<0.05, n=8)$. Consequently, the peak at $9.7 \mathrm{~Hz}$ in the PSD observed with the added leak was abolished once the synaptic current stimulus was introduced (supplemental Fig. 2B, available at www. jneurosci.org as supplemental material). Thus, the highfrequency oscillation formed in the presence of the artificial leak conductance was more sensitive to the synaptic stimulus than the control theta oscillation.

In summary, the addition of a leak conductance differentially affected the peak frequency of the PSD of the subthreshold and spike-train responses. In the subthreshold regime the amount of power was reduced without a shift in the peak frequency of the PSD. In the case of the spike-train response, the peak frequency in the PSD was shifted by $\sim 5 \mathrm{~Hz}$ without altering the amount of power at the peak. Adding a synaptic current-based stimulus in addition to the leak conductance, however, abolished the peak in the spike-train PSD.

\section{Role of spiking dynamics in the expression of theta oscillations}

The ability for an added leak conductance to differentially affect the oscillation frequency in the subthreshold and spiking regime suggested that the oscillatory dynamics in each situation oc- curred through different biophysical mechanisms. In stellate cells, the dynamics controlling the spike AHP could be important for the expression of theta oscillations in the spike train. Specifically, spike clustering at theta could be influenced by rebound dynamics driven by the AHP. Hence, the peak in the PSD could result from an increased probability of firing after a spike AHP, and not directly from subthreshold oscillatory behavior. In fact, previous modeling and experimental data in stellate cells suggests that the dynamics underlying the AHP are important in the formation of spike clusters (Fransen et al., 2004; Nolan et al., 2007). We hypothesized that changes in membrane conductance and time constant could potentially alter the AHP shape and rebound behavior in stellate cells, and thus alter the expression of theta oscillations in the spike train

To quantify the changes in the AHP of stellate cells, we averaged the membrane potential after the peak of the spike for a duration $0.3 \mathrm{~s}$. Because of small variations in spike threshold between cells, spikes were lined up at the voltage threshold for comparison. We took two measurements of the AHP: duration and amplitude. Duration was measured as the time from the trough of the AHP to the peak of the overshoot in membrane voltage after the AHP. In cases without an overshoot, the AHP duration was measured from the trough to the first crossing of baseline voltage (defined as the voltage at the end of the $0.3 \mathrm{~s}$ averaging window). Amplitude was measured as the voltage difference between the AHP trough and the end of the $0.3 \mathrm{~s}$ averaging window (Fig. 6A). Figure 6 shows the average shape of the AHP under each condition described.

We first quantified the AHP under control and with the added leak conductance (no synaptic stimuli). The duration of the AHP with the added leak conductance was significantly reduced compared with control (Fig. $6 A, B)(0.07 \pm 0.003$ vs $0.12 \pm 0.002, p<$ $0.05, n=7)$. The amplitude of the AHP, however, was similar under both conditions (Fig. $6 A, B)(3.9 \pm 0.3 \mathrm{mV}$ vs $4.2 \pm 0.4$ $\mathrm{mV}, p>0.05, n=7)$. Qualitatively, the AHP under both conditions had an overshoot after the trough (Fig. 6A). We quantified the overshoot by taking the difference in membrane voltage between the peak after the AHP and the membrane voltage at the end of the $0.3 \mathrm{~s}$ window. Under control, the overshoot was $2.08 \pm$ $0.31 \mathrm{mV}$, whereas with the added leak conductance the value decreased to $0.83 \pm 0.21 \mathrm{mV}(p<0.05, n=7)$. 
From the above results, we can account for the positive shift in the peak of the PSD observed with the addition of the leak conductance (Fig. 5B). By reducing the duration of the AHP relative to control, the leak conductance increases the firing frequency within a cluster. Hence, the peak in the PSD of the spike train represents the correlation between spikes at a given interval. The overshoot after the AHP provides a mechanism of correlating spikes at interspike intervals determined by the duration of the AHP.

We next quantified the AHP under three conditions involving synaptic stimuli. These included the following stimuli: synaptic conductance, synaptic current, and synaptic current plus the added leak conductance. With the synaptic conductance or synaptic current plus the added leak conductance the AHP was reduced both in terms of amplitude and duration compared with the situation when the cell was driven by the synaptic current stimulus alone (Fig. 6C-F) (one-way ANOVA, $p<0.05, n=8)$. With the synaptic current stimulus, the cell continued to support an overshoot in membrane voltage after the AHP (Fig. 6C) $(0.77 \pm 0.14 \mathrm{mV}, n=8)$, whereas with the synaptic conductance stimulus or the synaptic current stimulus plus the added leak conductance the overshoot was abolished (Fig. 6C,D, black traces). The absence of the overshoot in the presence of synaptic activity and an added leak conductance accounts for the absence of a peak in the PSD of the spike train under these conditions. Without the overshoot, the high correlation between spikes at theta intervals observed during spike clusters is lost.

Under conditions where the cells were driven by synaptic stimuli (conductance or current), the amplitude of the AHP was smaller than under control conditions or when the leak conductance was added without the synaptic stimuli (Fig. 6, compare $A, C, D)$. Hence, the AHP of cells driven by transient synaptic activity, rather than DC, was always smaller. We hypothesized that the reduction of the AHP with the synaptic stimuli is a result of spike generation at a lower mean membrane voltage compared with a DC stimulus. Maintaining a similar firing frequency as with DC under synaptic stimuli requires maintaining cells at a more hyperpolarized mean membrane voltage. The extra hyperpolarization reduces the driving force and activation level of the currents underlying the AHP and rebound dynamics. The relative difference in the magnitude of the hyperpolarizing currents underlying the AHP
A
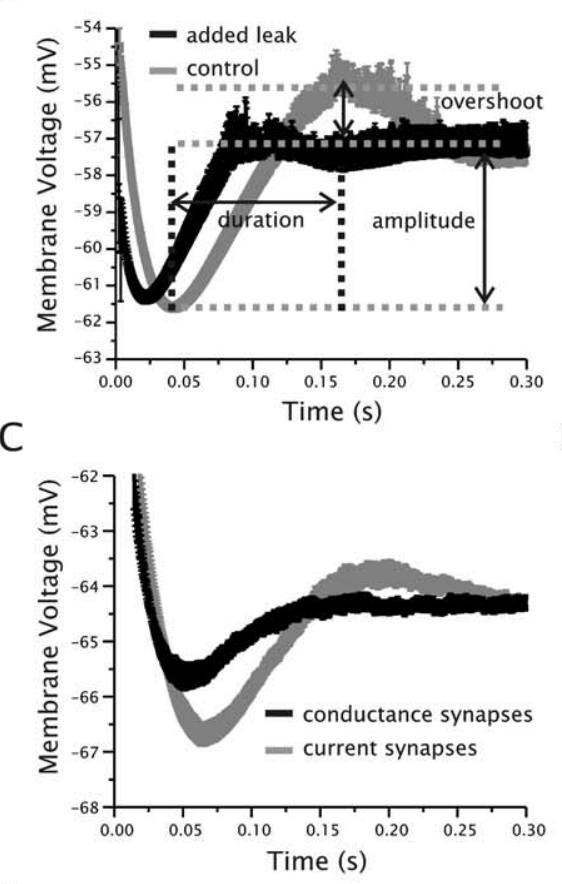

E

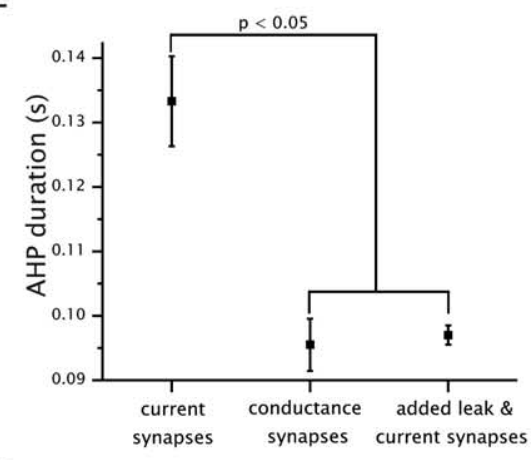

G

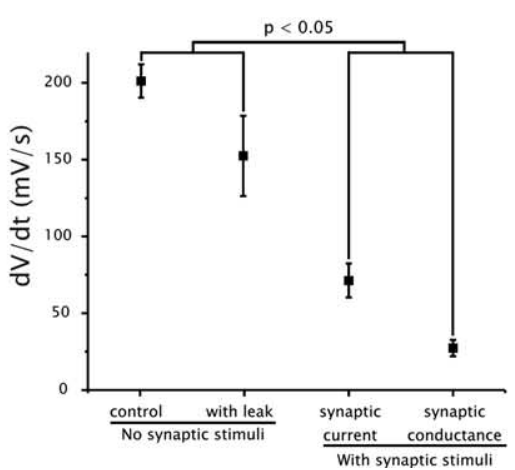

B

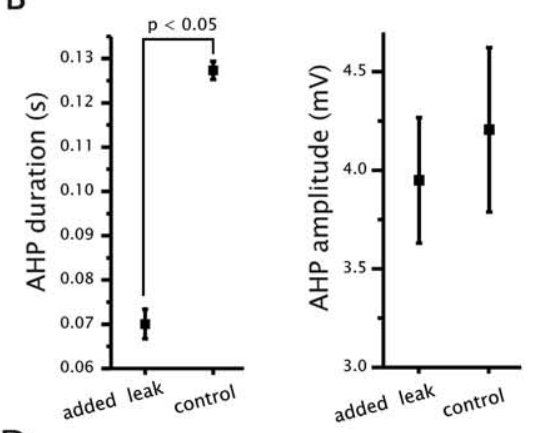

D

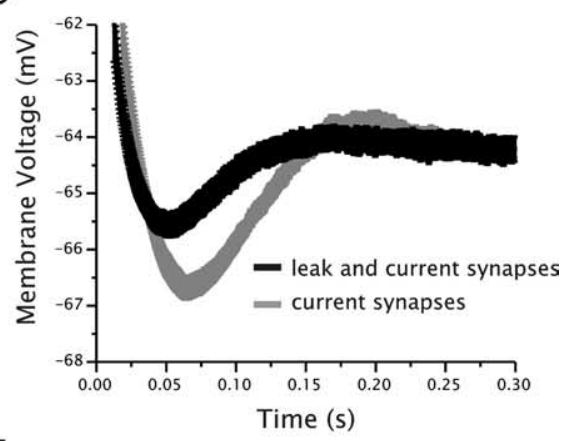

F

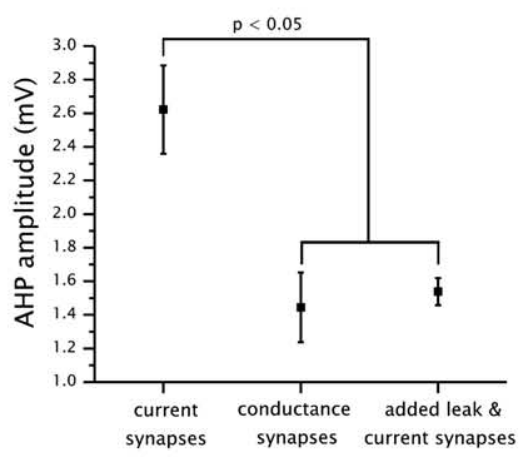

Figure 6. Conductance and synaptic-mediated membrane fluctuations alter the spike afterhyperpolarization (AHP) of stellate cells. $\boldsymbol{A}$, Average AHP shape under control (gray) and with added leak conductance (black). Line width denotes extent of error bars. $B$, Quantification of AHP duration (left) and amplitude (right). Note that the AHP duration, but not the amplitude, was significantly reduced by the presence of the added leak conductance. C, D, Average AHP shape under the synaptic current- (gray) and conductance-based (black) synapses. In D, the AHP under the synaptic current-based synapses (gray) is compared with the AHP under the added leak conductance plus the synaptic-current stimulus (black). Line widths denote extent of error bars. $\boldsymbol{E}, \boldsymbol{F}$, Quantification of AHP duration $(\boldsymbol{E})$ and amplitude $(\boldsymbol{F})$ under conditions described in $\boldsymbol{C}$ and $\boldsymbol{D}$. Note that both the duration and amplitude were affected by a change in conductance in the presence of synaptic stimuli. $\mathbf{G}$, Rate of change in membrane voltage $(d V / d t)$ between $0.01 \mathrm{~s}$ after the peak of the spike and the AHP trough under conditions described in $\boldsymbol{A}-\boldsymbol{D}$. Under synaptic stimuli (conductance or current) the $d V / d t$ value associated with the approach to the AHP trough was reduced compared with nonsynaptically driven cells. 
can be indirectly estimated by measuring the average rate of membrane voltage change associated with the approach to the AHP trough. Specifically, the faster the rate, the larger the relative current driving the voltage change. The rate in voltage change $(d V / d t)$ from $0.01 \mathrm{~s}$ after the peak of the spike to the AHP trough was larger in the control and leak modified cells than either of the hyperpolarized and synaptically driven conditions (Fig. 6G) (one-way ANOVA, $p<0.05, n=7$ ). These results indicate a reduced magnitude of the hyperpolarizing currents underlying the AHP under conditions were spiking is driven by synaptic activity. As a result, the AHP and corresponding rebound are more susceptible to the addition of a leak conductance than it would be if the cell was driven with DC stimuli at a more depolarized membrane potential. From this we can infer that the combination of synaptic-mediated fluctuations and increased membrane conductance abolished the expression of the peak in the PSD of the spike train by sufficiently altering the AHP in stellate cells.

Gauging the specific effects of the AHP requires uncoupling the reduction of the AHP from the corresponding changes observed in membrane input resistance, time constant, and subthreshold theta oscillations. We hypothesized that the expression of a peak at theta in the PSD of the spike train could be abolished with a reduction in the AHP, comparable in size to that under the conductance-based synaptic stimuli, without changing the input resistance or affecting the subthreshold oscillations.

The AHP in stellate cells is the product of numerous voltageand $\mathrm{Ca}^{2+}$-senstive $\mathrm{K}^{+}$currents. Application of apamine, cobalt, or cadmium blocks only a portion of the AHP in stellate cells (Khawaja et al., 2007). Hence, we used dynamic clamp to introduce a current $\left(I_{-\mathrm{AHP}}\right)$ that reduced the size of the AHP selectively without altering the subthreshold membrane dynamics (for details, see Materials and Methods) (Fig. 7 $A, B$ ). In essence, the $I_{- \text {AHP }}$ consisted of a negative $\mathrm{K}^{+}$current with a sharp and high voltage for activation, such that it was not active in the subthreshold range, but could be well recruited by a spike, allowing it to alter the AHP (Fig. 7A). Note the absence of the membrane overshoot after the AHP in the presence of $I_{- \text {AHP }}$ (Fig. $7 B$ ).

The principal qualitative effect of introducing $I_{-\mathrm{AHP}}$ in the absence synaptic stimuli was a marked reduction in spike clustering compared with control (Fig. 8A). Consequently, the PSD of spike trains with $I_{- \text {AHP }}$ had no observable peak at theta and significantly less power within this range compared with control (Fig. $8 B)(p<0.05, n=18)$. There was also significantly more power in the PSD at frequencies $<2.5 \mathrm{~Hz}(p<0.05, n=18)$. The lack of spike clustering also changed the distribution of ISI values such that the bimodality observed under control conditions was abolished in the presence of the $I_{- \text {AHP }}$ (Fig. $8 C$ ). We followed by measuring the PSD of spike trains of stellate cells in the presence of $I_{- \text {AHP }}$ and the synaptic current stimulus. As during the synaptic conductance stimulus, the PSD with $I_{- \text {AHP }}$ had significantly less power at theta and increased power at frequencies $<2.5 \mathrm{~Hz}$ (Fig. $8 E)(p<0.05, n=11)$. Thus, a reduction of the spike AHP was sufficient to remove the spike-train clustering and oscillatory behavior at theta without altering the subthreshold oscillations.

We followed by increasing the size of the AHP with dynamic clamp in the presence of the synaptic conductance stimulus. We reasoned that a larger AHP in the presence of the synaptic conductance stimulus could restore the peak at theta in the PSD of the spike train despite the conductance stimulus abolishing subthreshold oscillations. We used an inverted version of $I_{- \text {AHP }}$ $\left(I_{+A H P}\right)$ (see Materials and Methods) to generate an AHP of sufficient amplitude to support an overshoot in membrane voltage
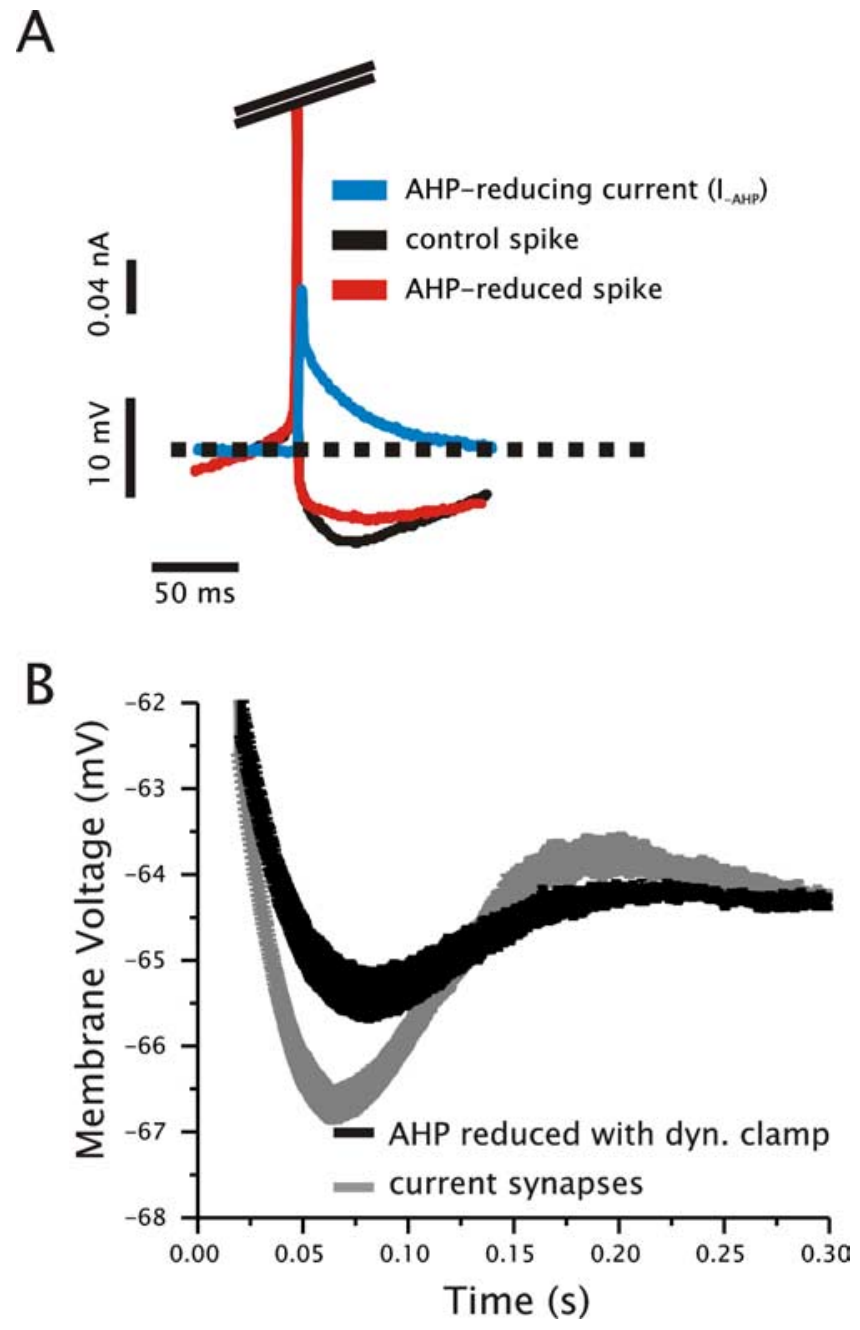

Figure 7. Reduction of stellate cell AHP using dynamic clamp. $\boldsymbol{A}$, Time course of control (black) and AHP-reduced spike (red), as well as the AHP-reducing current (blue; $I_{- \text {AHP }}$ ). $\boldsymbol{B}$, Average AHP shape in the presence of the synaptic current stimulus with (black) or without (gray) the artificially reduced AHP using $I_{-}$AHP. The line width denotes the extent of the error bars.

after the AHP trough in the presence of the synaptic conductance stimulus (Fig. 9B). We should note, however, that generating a peak in the PSD required a slightly larger AHP than with the synaptic current stimulus (Fig. 9B). This is likely because of the fact that the increased membrane conductance dampens the rebound and oscillatory dynamics involved in rebound firing. The presence of $I_{+A H P}$ restored the peak in the PSD of the spike train at theta (peak frequency at $6.2 \pm 0.3 \mathrm{~Hz}, n=8)($ Fig. 9C) and reduced the amount of power $<2.5 \mathrm{~Hz}$ (Fig. 9C) $(p<0.05, n=$ $8)$. These results substantiate our hypothesis that the AHP dynamics are critical for the expression of theta oscillations in the spike train of stellate cells.

\section{Discussion}

Using dynamic clamp we have shown that synaptic conductance and current stimuli produce distinct outputs in MEC stellate cells. Theta oscillations observed in the subthreshold membrane voltage and spike train were either abolished or significantly reduced in the presence of a synaptic conductance-based stimulus. Overall, our study is consistent with previous work in other systems that have shown that spike output can be fundamentally altered by synaptic conductance activity (Wolfart et al., 2005). 
A

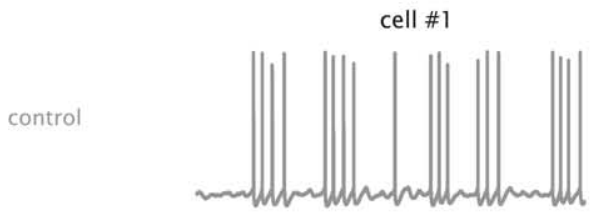

cell \#

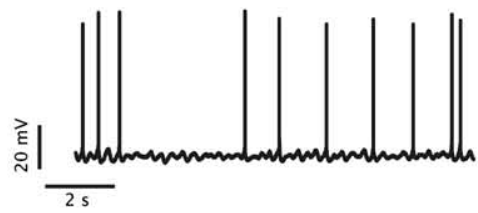

cell \#2

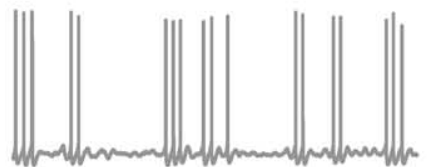

cell \#3

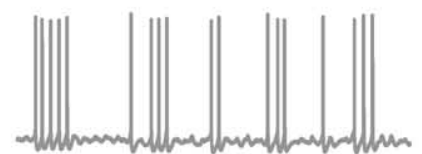

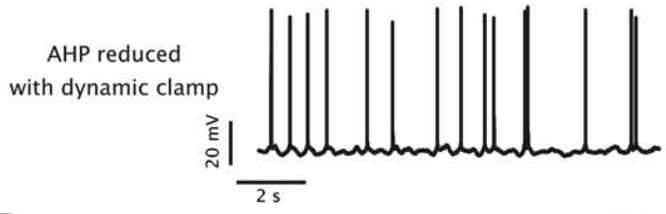

B

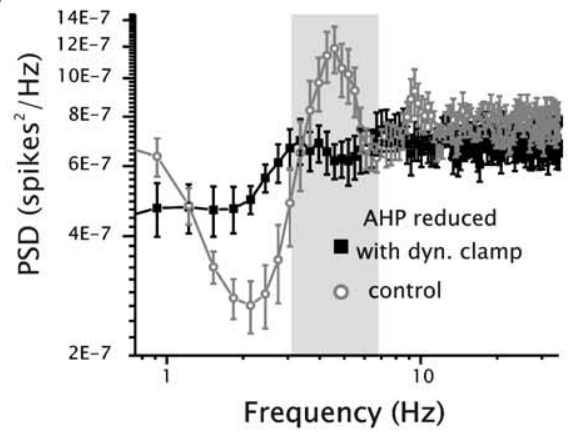

C

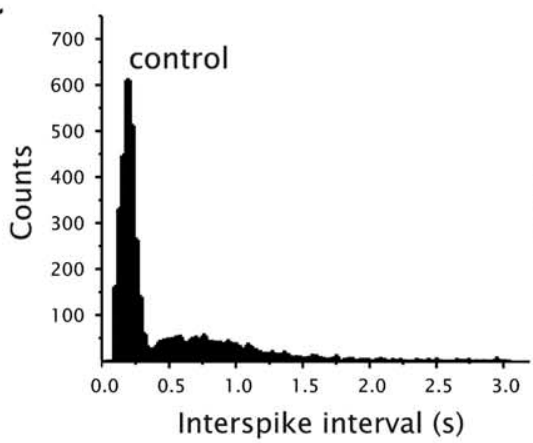

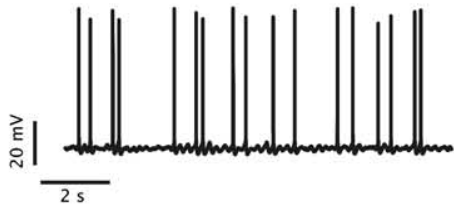

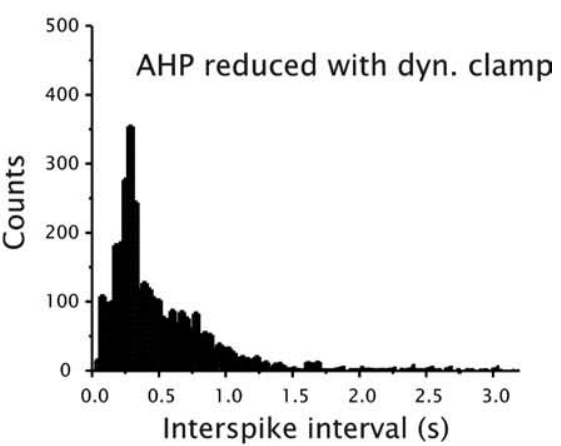

E
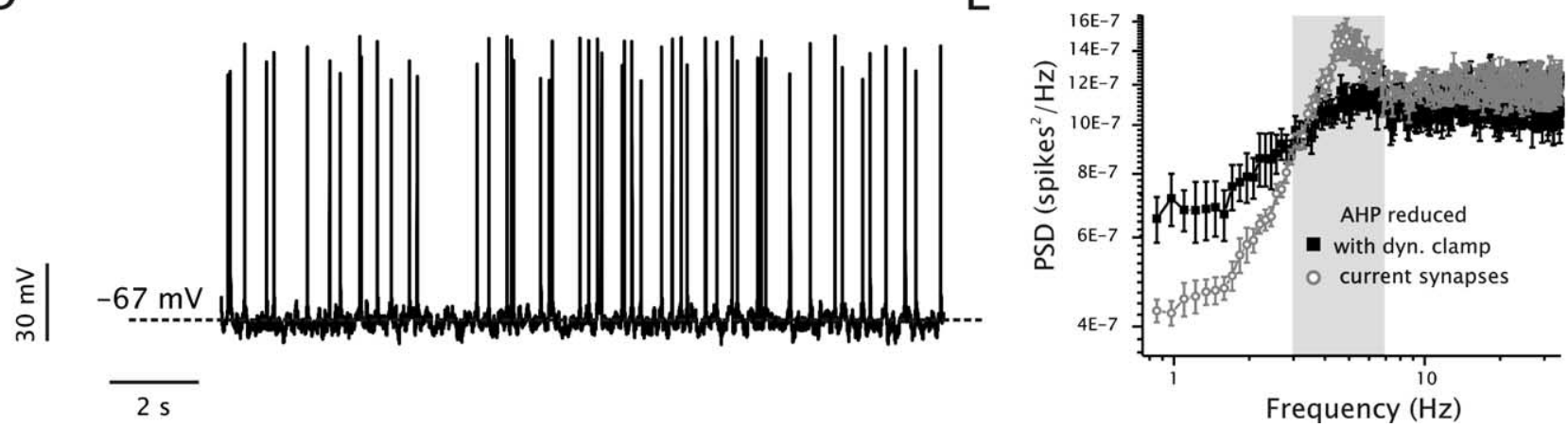

Figure 8. Reduction of stellate cell AHP abolishes theta oscillations expressed in the spike train. $A$, Representative examples of stellate cell membrane voltage traces under control (gray) and with the AHP-reducing current (black). B, Average spike-train PSD under control (gray circles) and in the presence of the AHP-reducing current (black squares). Note that the spike-train PSD in the presence of the AHP-reducing current has no peak at theta and significantly increased power at low frequencies $(0-2.5 \mathrm{~Hz})$. C, Spike time histograms of data in $\boldsymbol{B}$ during control (left) and in the presence of the AHP-reducing current (right). In the control situation, the histogram has two distinctive peaks, whereas with the AHP-reducing current there is only one. $\boldsymbol{D}$, Representative example of a stellate cell membrane voltage trace in the presence of both a synaptic current stimulus and the AHP-reducing current. $E$, Average spike-train PSD under a synaptic current stimulus (gray circles) and in the presence of both the AHP-reducing and synaptic current stimulus (black squares).

\section{Biophysical mechanisms involved in the expression of theta oscillations}

The mechanism by which the synaptic conductance stimulus eliminated the expression of theta oscillations differed between the subthreshold and spiking regimes. Although a drop in membrane input resistance was involved under both situations, it affected each regime in a distinct manner. In the subthreshold situation, the decrease in membrane input resistance dampened the subthreshold oscillations by reducing the resonance properties of the membrane as well as the size of the $I_{\mathrm{H}}$-mediated membrane sag. Although the addition of a leak conductance was sufficient to dramatically decrease the amplitude of the subthreshold oscillations, it did not alter the frequency at which the oscillation occurred. Assuming that the oscillation dynamics can be described by the linear properties of the membrane, and hence the resonant properties of the membrane, this result can be accounted for by a parallel inductive circuit, in which case a change in resistance significantly alters the amplitude of the resonance, but can have a very small effect on the resonant frequency (Hutcheon et al., 1996; Erchova et al., 2004).

The expression of theta oscillations in the spike train depended on the size of the stellate cell AHP and its interaction with membrane noise. The control AHP was reliably followed by an overshoot in membrane voltage. This feature was absent in the presence of a synaptic conductance stimulus. The overshoot is responsible for increasing the probability that another spike will be generated at an ISI value equal to theta.

In the absence of synaptic noise, the addition of a leak conductance alone did not abolish spike clustering. In these conditions, the intracluster ISI values decreased, which produced a 
A

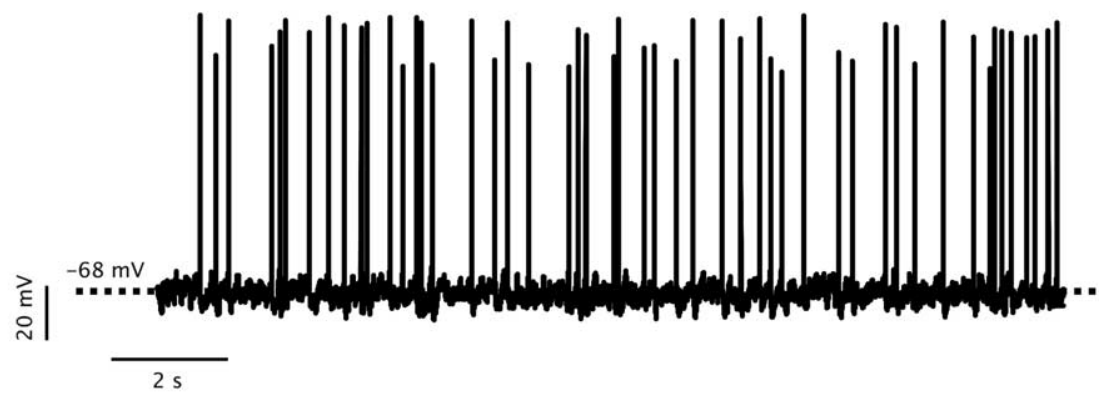

B

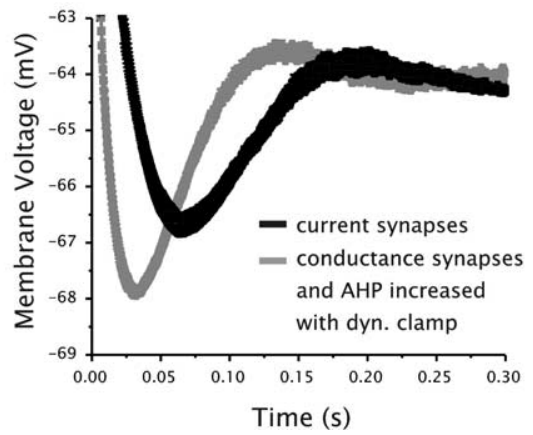

C

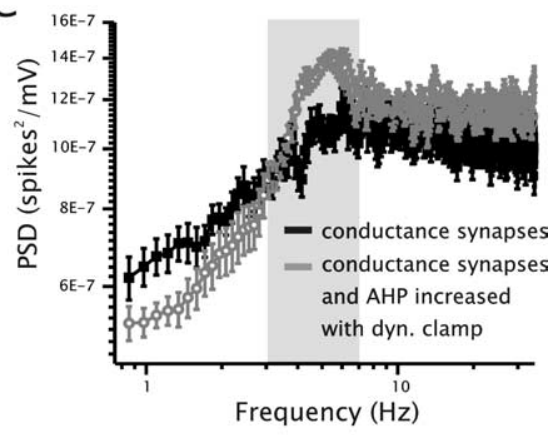

Figure 9. Increasing the AHP in stellate cells restores the expression of theta oscillations in the spike train. $\boldsymbol{A}$, Representative example of a stellate cell membrane voltage trace in the presence of both a synaptic conductance stimulus and the AHP-increasing current. $\boldsymbol{B}$, Average AHP shape in the presence of the synaptic current stimulus with (gray) or without (black) the artificially

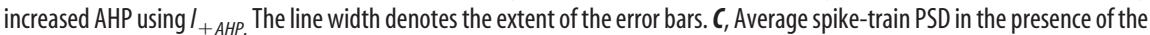
synaptic conductance stimulus with (gray circles) or without (black squares) the artificially increased AHP using $I_{+}$AHP.

shift in the peak of the PSD of the spike train from $4.9 \mathrm{~Hz}$ to 9.7 $\mathrm{Hz}$ (Fig. 6B). This result differed markedly from the subthreshold oscillations, where a shift in peak frequency was not observed with the added leak conductance (Fig. 5B). The differential response of the subthreshold and spike-train oscillatory behavior to an increase in leak conductance originates from the fact that the biophysics underlying the AHP are different from those sustaining the subthreshold oscillations. In particular, the time between spikes will be influenced by the membrane time constant, which, in turn, can be significantly altered by a change in membrane resistance. The introduction of a leak conductance changes the membrane time constant and the spike frequency within clusters by shortening the duration of the AHP, but does not alter the frequency of the subthreshold oscillations or resonance. This also suggests that the final duration of the AHP is heavily influenced by the passive properties of the membrane and that the AHP outlasts the active $\mathrm{K}^{+}$conductances initially responsible for generating it.

The amplitude of the AHP was always smaller when spikes were driven with synaptic-mediated fluctuations (i.e., noise). This results from the fact that under noisy conditions the cell must be held at a more negative mean membrane voltage to maintain the same firing frequency as the control condition (Barbi et al., 2000; Longtin, 2000; Chance et al., 2002; Miller and Troyer, 2002). The fluctuating nature of the synaptic stimulus protocol permits occasional excursions past spike threshold that would not be possible if the cells were held at the same mean voltage under quiescent conditions. In the present study, we show that the generation of spikes at a lower mean membrane voltage can reduce the spike AHP. By generating spikes at a lower mean membrane voltage the AHP is sufficiently reduced such that an increase in membrane conductance can eliminate the increased probability of generating a second spike at a theta interval.

Reducing the AHP directly without affecting subthreshold dynamics abolished the peak in the PSD (Fig. 8). This signifies that the AHP, and not the subthreshold oscillations, are directly responsible for the expression of theta in the spike train. In stellate cells the amplitude of the oscillations are highly variable. The variability stems from the fact that intrinsic noise sources originating from $\mathrm{Na}^{+}$channel fluctuations are involved in their generation (White et al., 1998; Dorval and White, 2005). Consequently, with a reduced AHP and the absence of an overshoot there is no assurance that when a spike is generated another will follow with an interval equal to theta. Hence, spikes in stellate cells cannot be interpreted as events "riding" the subthreshold membrane voltage trajectory. If this were the case one would predict that spikes would skip theta cycles randomly and produce no significant power at theta in the spike train. From this we can conclude that spike clustering does not represent the entrainment of regular spike firing by subthreshold oscillations as has been suggested previously (Alonso and Klink, 1993).

\section{Implications for network behavior and in vivo activity}

Our results suggest that the expression of oscillatory properties in stellate cells have the potential to be heavily modulated, or even eliminated, in vivo. This does not, however, imply that intrinsic cell properties are inconsequential. It is possible that synaptic background activity acts to modulate the expression of stellate cell oscillations. Given the possibility that distal synaptic inputs behave more like a current stimulus, the location of an input on a cell could be used to modulate the oscillatory nature of the output. Nevertheless, the elimination of theta oscillations by synaptic inputs has important implications for both the entorhinal cortex and hippocampus. Because stellate cells receive significant somatic input (Kohler, 1986; Swanson and Kohler, 1986; Gloor, 1997; Klink and Alonso, 1997), and only modest conductance changes were required to eliminate theta oscillations, it seems likely that the results presented in this study play a significant role in vivo.

To date, there have been no intracellular recordings of stellate cells in vivo to indicate that intrinsic oscillatory mechanisms play a significant role in shaping overall entorhinal cortical and hippocampal activity. The role of intrinsic stellate cell properties in network behavior has been assumed based on the fact that in vivo single-unit and field potential recordings from layer II in anesthetized animals show a peak in power at theta in the PSD (Mitchell and Ranck, 1980; Alonso and Garcia-Austt, 1987a,b) that matches the intrinsic frequency of stellate cell membrane oscillations (Alonso and Llinas, 1989). Although under anesthesia single units from superficial layers of the entorhinal cortex show both a peak in the PSD and a mean firing rate that is modulated at theta (Alonso and Garcia-Austt, 1987b; Dickson et al., 
1995), similar recordings from freely behaving animals show no peak in the PSD, but like other cells in the hippocampus and deep entorhinal cortex, continue to have their mean firing rate modulated at theta (Frank et al., 2001). Given the prominence of theta oscillations in the spike-train response in our recordings under control and current-based stimulus, it would be expected that if intrinsic oscillatory dynamics were dominant under in vivo conditions the PSD of single units would consistently show a peak at theta.

Recordings from the entorhinal cortex of freely behaving animals have shown that cells from superficial layers support the expression of hexagonal grid cell fields (Hafting et al., 2005). Modeling studies have implicated intrinsic subthreshold oscillations in stellate cells in the formation of grid cell fields (O'Keefe and Burgess, 2005; Burgess et al., 2007). It has been proposed that beat frequencies generated by the interference between intrinsic somatic and dendritic oscillations provides the basis for the construction of a grid cell field (Burgess et al., 2007). Furthermore, intracellular data indicating a gradient in the subthreshold oscillation frequency of stellate cells has been implicated in establishing the corresponding gradient in the spacing between grid cells observed along the dorsal-ventral axis of the entorhinal cortex (Giocomo et al., 2007). An assumption in this model has been that spikes have no contribution to the expression of subthreshold theta oscillations in the spike train. Although our data suggests that relatively modest conductance increases and realistic noise levels can abolish theta oscillations, the exact contribution of intrinsic properties under in vivo conditions is difficult to precisely ascertain. However, our data clearly shows that the spiking dynamics through the AHP are critical for the expression of theta in the spike train. Consequently, properties observed in the subthreshold oscillation, such as the gradient in frequency along the dorsal-ventral axis, cannot be assumed to be translated into the spiking regime of stellate cells. Our data suggests that drawing connections between single cell properties and their extension into network behavior requires considering both subthreshold and spiking dynamics under more realistic in vivo conditions.

\section{References}

Alonso A, Garcia-Austt E (1987a) Neuronal sources of theta rhythm in the entorhinal cortex of the rat. I. Laminar distribution of theta field potentials. Exp Brain Res 67:493-501.

Alonso A, Garcia-Austt E (1987b) Neuronal sources of theta rhythm in the entorhinal cortex of the rat. II. Phase relations between unit discharges and theta field potentials. Exp Brain Res 67:502-509.

Alonso A, Klink R (1993) Differential electroresponsiveness of stellate and pyramidal-like cells of medial entorhinal cortex layer II. J Neurophysiol 70:128-143.

Alonso A, Llinas RR (1989) Subthreshold $\mathrm{Na}^{+}$-dependent theta-like rhythmicity in stellate cells of entorhinal cortex layer II. Nature 342:175-177.

Anderson JS, Lampl I, Gillespie DC, Ferster D (2000) The contribution of noise to contrast invariance of orientation tuning in cat visual cortex. Science 290:1968-1972.

Bair W, Koch C, Newsome W, Britten K (1994) Power spectrum analysis of bursting cells in area MT in the behaving monkey. J Neurosci 14:2870-2892.

Barbi M, Chillemi S, Di Garbo A (2000) The leaky integrate-and-fire with noise: a useful tool to investigate SR. Chaos Solitons Fractals 11:1849-1853.

Berg RW, Alaburda A, Hounsgaard J (2007) Balanced inhibition and excitation drive spike activity in spinal half-centers. Science 315:390-393.

Bernander O, Douglas RJ, Martin KA, Koch C (1991) Synaptic background activity influences spatiotemporal integration in single pyramidal cells. Proc Natl Acad Sci USA 88:11569-11573.

Berretta N, Jones RS (1996) A comparison of spontaneous EPSCs in layer II and layer IV-V neurons of the rat entorhinal cortex in vitro. J Neurophysiol 76:1089-1100.
Borg-Graham LJ, Monier C, Fregnac Y (1998) Visual input evokes transient and strong shunting inhibition in visual cortical neurons. Nature 393:369-373.

Burgess N, Barry C, O’Keefe J (2007) An oscillatory interference model of grid cell firing. Hippocampus 17:801-812.

Chance FS, Abbott LF, Reyes AD (2002) Gain modulation from background synaptic input. Neuron 35:773-782.

Destexhe A, Pare D (1999) Impact of network activity on the integrative properties of neocortical pyramidal neurons in vivo. J Neurophysiol 81:1531-1547.

Destexhe A, Rudolph M, Fellous JM, Sejnowski TJ (2001) Fluctuating synaptic conductances recreate in vivo-like activity in neocortical neurons. Neuroscience 107:13-24.

Destexhe A, Rudolph M, Pare D (2003) The high-conductance state of neocortical neurons in vivo. Nat Rev Neurosci 4:739-751.

Dickson CT, Kirk IJ, Oddie SD, Bland BH (1995) Classification of thetarelated cells in the entorhinal cortex: cell discharges are controlled by the ascending brainstem synchronizing pathway in parallel with hippocampal theta-related cells. Hippocampus 5:306-319.

Dorval Jr AD, White JA (2005) Channel noise is essential for perithreshold oscillations in entorhinal stellate neurons. J Neurosci 25:10025-10028.

Dorval AD, Christini DJ, White JA (2001) Real-time linux dynamic clamp: a fast and flexible way to construct virtual ion channels in living cells. Ann Biomed Eng 29:897-907.

Erchova I, Kreck G, Heinemann U, Herz AV (2004) Dynamics of rat entorhinal cortex layer II and III cells: characteristics of membrane potential resonance at rest predict oscillation properties near threshold. J Physiol (Lond) 560:89-110.

Frank LM, Brown EN, Wilson MA (2001) A comparison of the firing properties of putative excitatory and inhibitory neurons from CA1 and the entorhinal cortex. J Neurophysiol 86:2029-2040.

Fransen E, Alonso AA, Dickson CT, Magistretti J, Hasselmo ME (2004) Ionic mechanisms in the generation of subthreshold oscillations and action potential clustering in entorhinal layer II stellate neurons. Hippocampus 14:368-384.

Giocomo LM, Zilli EA, Fransen E, Hasselmo ME (2007) Temporal frequency of subthreshold oscillations scales with entorhinal grid cell field spacing. Science 315:1719-1722.

Gloor P (1997) The temporal lobe and limbic system. New York: Oxford UP.

Hafting T, Fyhn M, Molden S, Moser MB, Moser EI (2005) Microstructure of a spatial map in the entorhinal cortex. Nature 436:801-806.

Harsch A, Robinson HP (2000) Postsynaptic variability of firing in rat cortical neurons: the roles of input synchronization and synaptic NMDA receptor conductance. J Neurosci 20:6181-6192.

Hutcheon B, Miura RM, Puil E (1996) Models of subthreshold membrane resonance in neocortical neurons. J Neurophysiol 76:698-714.

Jones RS (1994) Synaptic and intrinsic properties of neurons of origin of the perforant path in layer II of the rat entorhinal cortex in vitro. Hippocampus 4:335-353.

Jones RS, Woodhall GL (2005) Background synaptic activity in rat entorhinal cortical neurones: differential control of transmitter release by presynaptic receptors. J Physiol (Lond) 562:107-120.

Khawaja FA, Alonso AA, Bourque CW (2007) $\mathrm{Ca}^{2+}$-dependent $\mathrm{K}^{+}$currents and spike-frequency adaptation in medial entorhinal cortex layer II stellate cells. Hippocampus 17:1143-1148.

Klink R, Alonso A (1993) Ionic mechanisms for the subthreshold oscillations and differential electroresponsiveness of medial entorhinal cortex layer II neurons. J Neurophysiol 70:144-157.

Klink R, Alonso A (1997) Morphological characteristics of layer II projection neurons in the rat medial entorhinal cortex. Hippocampus 7:571-583.

Koch C (1999) Biophysics of computation: information processing in single neurons. New York: Oxford UP.

Kohler C (1986) Intrinsic connections of the retrohippocampal region in the rat brain. II. The medial entorhinal area. J Comp Neurol 246:149-169.

Longtin A (2000) Effect of noise on the tuning properties of excitable systems. Chaos Solitons Fractals 11:1835-1848.

Miller KD, Troyer TW (2002) Neural noise can explain expansive, powerlaw nonlinearities in neural response functions. J Neurophysiol $87: 653-659$. 
Mitchell SJ, Ranck Jr JB (1980) Generation of theta rhythm in medial entorhinal cortex of freely moving rats. Brain Res 189:49-66.

Netoff TI, Banks MI, Dorval AD, Acker CD, Haas JS, Kopell N, White JA (2005) Synchronization in hybrid neuronal networks of the hippocampal formation. J Neurophysiol 93:1197-1208.

Nolan MF, Dudman JT, Dodson PD, Santoro B (2007) HCN1 channels control resting and active integrative properties of stellate cells from layer II of the entorhinal cortex. J Neurosci 27:12440-12451.

O'Keefe J, Burgess N (2005) Dual phase and rate coding in hippocampal place cells: theoretical significance and relationship to entorhinal grid cells. Hippocampus 15:853-866.

Pare D, Shink E, Gaudreau H, Destexhe A, Lang EJ (1998) Impact of spontaneous synaptic activity on the resting properties of cat neocortical pyramidal neurons In vivo. J Neurophysiol 79:1450-1460.

Shadlen MN, Newsome WT (1998) The variable discharge of cortical neu- rons: implications for connectivity, computation, and information coding. J Neurosci 18:3870-3896.

Softky WR, Koch C (1993) The highly irregular firing of cortical cells is inconsistent with temporal integration of random EPSPs. J Neurosci 13:334-350.

Swanson LW, Kohler C (1986) Anatomical evidence for direct projections from the entorhinal area to the entire cortical mantle in the rat. J Neurosci 6:3010-3023.

van Vreeswijk C, Sompolinsky H (1996) Chaos in neuronal networks with balanced excitatory and inhibitory activity. Science 274:1724-1726.

White JA, Klink R, Alonso A, Kay AR (1998) Noise from voltage-gated ion channels may influence neuronal dynamics in the entorhinal cortex. J Neurophysiol 80:262-269.

Wolfart J, Debay D, Le Masson G, Destexhe A, Bal T (2005) Synaptic background activity controls spike transfer from thalamus to cortex. Nat Neurosci 8:1760-1767. 